\title{
Interrogating glioma-associated microglia/macrophage dynamics under CSF-1R therapy with multi-tracer in vivo PET/MR imaging
}

Claudia Foray ${ }^{1,2^{*}}$, Cristina Barca ${ }^{1,2^{*}}$, Alexandra Winkeler ${ }^{3}$, Stefan Wagner ${ }^{4}$, Sven Hermann ${ }^{1}$, Michael Schäfers ${ }^{1,4}$, Oliver M. Grauer ${ }^{5 \dagger}$, Bastian Zinnhardt ${ }^{1,2,4,6^{+}}$, Andreas H. Jacobs ${ }^{1,2,7^{\dagger}}$

${ }^{1}$ European Institute for Molecular Imaging (EIMI), University of Münster, Münster, Germany

${ }^{2}$ PET Imaging in Drug Design and Development (PET3D)

${ }^{3}$ Université Paris-Saclay, CEA, CNRS, Inserm, BioMaps, Orsay, France

${ }^{4}$ Department of Nuclear Medicine, University Hospital Münster, Münster, Germany

${ }^{5}$ Department of Neurology, University Hospital Münster, Münster, Germany

${ }^{6}$ Biomarkers \& Translational Technologies (BTT), Neurosciences and rare diseases (NRD), Pharma Research

\& Early Development (pRED), F. Hoffmann-La Roche Ltd., Basel, Switzerland

${ }^{7}$ Department of Geriatrics with Neurology, Johanniter Hospital, and Centre for integrated oncology (CIO)

of the University Hospital Bonn, Bonn, Germany

${ }^{*}$ Contributed equally to this work

${ }^{\dagger}$ Equal contribution

Corresponding authors: Andreas H. Jacobs and Claudia Foray (Postdoc)

European Institute for Molecular Imaging,

University of Münster

Waldeyerstr. 15

D-48149 Münster

Tel. +492518349334

Fax. +492518349313

ahjacobs@uni-muenster.de; claudia.foray@uni-muenster.de;

Funding

This work has received support from the EU 7th Framework Programme (FP7/2007-2013) under grant agreement $n^{\circ} 278850$ (INMiND), the Horizon2020 Programme under grant agreement $n^{\circ} 675417$ (PET3D), the EU/EFPIA/Innovative Medicines Initiative 2 Joint Undertaking (Immune-Image GA831514) under grant agreement $n^{\circ}$ 831514, the 'Cells-in-Motion' Cluster of Excellence (CiM) Graduate School, by the Interdisciplinary Center for Clinical Research (IZKF core unit PIX), Münster, Germany, and by the HerbertWorch-Foundation, Bonn, Germany.

Short title: CSF-1R-based GAMM modulation in glioma

Word count: 4997

Immediate Open Access: Creative Commons Attribution 4.0 International License (CC BY) allows users to share and adapt with attribution, excluding materials credited to previous publications.

License: https://creativecommons.org/licenses/by/4.0/.

Details: https://inm.snmjournals.org/page/permissions.

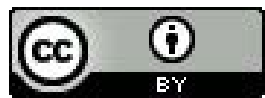




\section{ABSTRACT}

Background: Glioma-associated microglia/macrophages (GAMM) are key players in creating an immunosuppressive microenvironment. They can be efficiently targeted by inhibiting the colony stimulating factor-1 receptor (CSF-1R). We employed non-invasive PET/CT-MRI using 18F-FET (amino-acid metabolism) and 18F-DPA-714 (translocator protein - TSPO) to (i) understand the role of GAMM in glioma initiation, (ii) monitor in-vivo therapy-induced GAMM depletion, and (iii) observe GAMM repopulation after drug withdrawal.

Methods: C57BL/6 mice $(n=44)$ orthotopically implanted with syngeneic mouse GL261 glioma cells were treated with different regimens using PLX5622 (CSF-1R inhibitor) or vehicle, establishing a "preconditioning" and a "repopulation" model, respectively. Mice underwent longitudinal PET/CT-MR imaging.

Results: The preconditioning model (PM) indicated similar tumor growth based on MRI (44.5 $\pm 24.8 \%)$, 18F-FET- (18.3 $\pm 11.3 \%)$ and 18F-DPA-714-PET (16 $\pm 19.04 \%)$ volume dynamics in all groups, suggesting that GAMM are not involved in glioma initiation. The repopulation-model (RM) showed (i) significantly reduced 18F-DPA-714 uptake $(-45.6 \pm 18.4 \%)$ together with (ii) significantly reduced GAMM infiltration even after repopulation, and (iii) a significantly decreased tumor volume (-54.29 $\pm 8.6 \%)$ with repopulation as measured by MRI, supported by a significant reduction of $18 \mathrm{~F}-\mathrm{FET}$ uptake (-50.2 $\pm 5.3 \%)$.

Conclusion: 18F-FET- and 18F-DPA-714-PET/MRI allow the non-invasive assessment of glioma growth under various regimens of CSF-1R therapy. CSF-1R-mediated modulation of GAMM may be of high interest as (co-)therapy against glioma.

Keywords: glioma, GAMM, 18F-DPA-714, TSPO, CSF-1R, C57BL/6 


\section{INTRODUCTION}

The aggressiveness and molecular complexity of glioblastoma multiforme challenges the current standard care therapy, limiting the median overall survival to 14-16 months (1). In the last decades, new immunotherapeutic strategies have been developed for gliomas. Despite some promising results, there are currently no approved immunotherapies that proved to be efficient against glioblastoma multiforme (2). The high heterogeneity of the tumor microenvironment (TME) plays an important role in therapy resistance, and glioma-associated myeloid cells, such as glioma-associated microglia/macrophages (GAMM), monocytes, and myeloid-derived suppressor cells (MDSCs) (including polymorphonuclear (PMN) and monocytic (M-)MDSCs) are key players in the establishment of an immunosuppressive environment which favors glioma immune evasion and progression (3). Moreover, chronically activated resident immune cells exacerbate the inflammatory response, leading to a high state of neuroinflammation, while participating in the development of an immunosuppressive TME (4). Therefore, targeting gliomaassociated myeloid cells represents an important strategy to develop new glioma microenvironmenttargeted therapies. These cell populations are dependent on colony stimulating factor-1 receptor (CSF-1R) signaling for their survival (5). Small molecule CSF-1R inhibitors are used to study the dynamics of these cells in glioma progression and glioma-associated inflammation profiting from their ability to modulate GAMM through a mechanism of depletion and repopulation.

Magnetic resonance imaging (MRI) and amino acid PET imaging with methyl-11C-methionine (11CMET) or 18F-fluoro-ethyl-tyrosine (18F-FET, amino acid transport) are part of the clinical imaging routine to diagnose and follow-up patients with gliomas (6-9). The use of 18F-FET has been reported to have a major clinical value in providing important information for tumor delineation and differentiation, and the assessment of post-therapeutic modifications and relapses (10). 18F-FET-PET is highly specific for glioma tissue but it falls short in visualizing the reactive and infiltrating myeloid component of the glioma microenvironment (11). Other radiotracers, including the $18 \mathrm{kDa}$ translocator protein (TSPO) targeting PET tracer $N, N$-diethyl-2-[4-(2-18F-fluoroethoxy)phenyl]-5,7-dimethylpyrazolo[1,5-a]pyrimidine-3-acetamide 
(18F-DPA-714) have been employed to visualize the myeloid cell compartment in gliomas (12). 18F-DPA714-PET has been reported to give complementary information to 18F-FET especially with regards to resident and infiltrating immune cells in pre-clinical glioma models as well as in patients $(13,14)$. As the timing of immunomodulation may be critical for therapy outcome, non-invasive imaging tools are highly useful in the assessment of the glioma microenvironment before, during and after therapeutic intervention with novel immunomodulatory compounds (15).

Here, we performed a multi-modal, dual-tracer imaging study in a syngeneic mouse model of glioma employing 18F-FET- and 18F-DPA-714-PET using a CSF-1R inhibitor to investigate the interplay between glioma progression and related immune cell dynamics. Performing a preconditioning study, we aimed to investigate the possible role of GAMM in glioma initiation. In the repopulation model, we aimed to assess the therapeutic effect of CSF-1R inhibition-induced depletion and subsequent repopulation on GAMM dynamics in an established tumor.

We hypothesized that a GAMM depletion-repopulation approach would be beneficial, resulting in reduced tumor size and decreased neuroinflammation, and that 18F-DPA-714 is a suitable imaging readout to in vivo investigate GAMM dynamics during the course of CSF-1R inhibitor therapy. Taking advantage of the reversible inhibitory effect of the drug, we were able to monitor non-invasively the dynamics during CSF-1R inhibitor-mediated GAMM depletion and repopulation. 


\section{MATERIALS AND METHODS}

\section{Cell Culture}

Mouse GL261 glioma cells were cultured in T-75 cell culture flasks as an adherent monolayer in DMEM supplemented with GlutaMax, $10 \%$ heat-inactivated FCS and $1 \%$ penicillin/streptomycin at $37{ }^{\circ} \mathrm{C}$ in a humidified incubator maintained at $5 \% \mathrm{CO}_{2}$ prior to intracranial implantation.

\section{Study Approval}

All experiments were conducted in accordance with the German Law on the Care and Use of Laboratory Animals and approved by the Landesamt für Natur, Umwelt und Verbraucherschutz (LANUV) of North Rhine-Westphalia and the ARRIVE guidelines (16).

\section{Study Design}

In total, $\mathrm{n}=51 \mathrm{C} 57 \mathrm{BL} / 6$ female mice of $8-10$ weeks of age were orthotopically implanted (intra-striatal injection, coordinates in relation to bregma: lateral $-2.0 \mathrm{~mm}$, anterior-posterior $+0.5 \mathrm{~mm}$, dorsal-ventral $3.0 \mathrm{~mm}$ ) with $2 \times 10^{5}$ mouse GL261 cells in $2 \mu \mathrm{I} \mathrm{NaCl} \mathrm{0.9 \% .} \mathrm{During} \mathrm{all} \mathrm{experimental} \mathrm{procedures,} \mathrm{mice} \mathrm{were}$ anesthetized with $1.5 \%$ to $2 \%$ isoflurane (Abbott Animal Health) in $100 \%$ O2. After surgery the animals were weighted for at least three days and before each imaging session to monitor their health condition (Supplemental Figure 1). Applying different regimens of CSF-1R inhibitor the study was divided into two parts, a preconditioning model (PM) where mice were treated with CSF-1R inhibitor 14 days prior to tumor implantation, and a repopulation model (RM) in which acute depletion and repopulation of microglial cells were performed. The mice underwent the imaging protocol of sequential gadolinium-contrast enhanced (Gd-CE) T1w-MRI, and 18F-FET- and 18F-DPA-714-PET-CT as described previously (17). Full details are available in the Supplemental M\&M, Figure $1 \mathrm{~A}$ and Figure 4A.

\section{MRI Studies}

MRI was conducted for 3D-localisation of the glioma and for co-registration with PET/CT images. The mice were anaesthetized and the lateral tail vein was cannulated using a 26 Ga catheter. A T1 SE 2D 
sequence was acquired in a $1 \mathrm{~T}$ nanoScan PET/MRI scanner equipped with a MH20 coil (Mediso Medical Imaging Systems, resolution: $0.27 \times 0.27 \times 0.9 \mathrm{~mm}$ ). Gadolinium-contrast agent (Gadovist, Bayer Pharmaceutical) was injected via the catheter $(50 \mu \mathrm{mol} / \mathrm{kg})$ and a post-Gd T1 sequence was acquired.

\section{PET Studies}

PET images were acquired on a high-resolution small animal PET scanner (32 module quadHIDAC, Oxford Positron Systems Ltd.) with uniform spatial resolution (<1 mm FWHM). 18F-DPA-714 PET images were acquired 60-80 minutes post intravenous (i.v.) injection of $14.3 \pm 2.6 \mathrm{MBq}$ 18F-DPA-714. 18F-FET PET images were acquired 20-30 minutes post i.v. injection of $10.6 \pm 0.6 \mathrm{MBq}$ of 18F-FET. See Supplementary M\&M for further details.

\section{Volumetric Analysis}

Imaging data were analyzed using the in-house developed software MEDgical as described

previously (17). Briefly, an atlas-based right hemisphere volume-of-interest (VOI) was threshold to delineate tumor and tracer uptake volumes after co-registration of PET/CT scans with MR images. Tumorto-background ratios (T/B) were calculated.

\section{Immunoreactivity}

After the last imaging examination, mice were sacrificed and perfused with $0.9 \% \mathrm{NaCl}$ and $4 \%$ PFA. Brains were fixed overnight in 4\% PFA, embedded in paraffin, cut in coronal sections and processed as previously described (17). To characterize the TME and therapy-induced modifications, CSF-1R/Iba1 and TSPO/Iba1 dual-labeling with fluorescent antibodies was performed in PM and RM studies. Quantification of positive cells were made in biological triplicates counting manually or using the bioimage analysis software QuPath (https://qupath.github.io) (18). Antibodies are available in Supplemental Table 1.

\section{Multiparametric Flow Cytometry (FACS)}

Myeloid-derived cells were isolated after processing of the tissues as described before (17). The cells were stained with a panel of directly labeled monoclonal antibodies (mAbs) (Supplemental Table 2). 
All samples were analyzed using the NaviosTM flow cytometer and the Kaluza 2.1 Software (Beckman Coulter, Krefeld, Germany). See Supplemental M\&M for additional data.

\section{Statistical Analysis}

Statistical analysis was performed using Prism 6 (GraphPad Software, Inc., USA). Differences over time in radiotracer uptake ratios and tracer uptake volumes intra- and inter-groups were tested using either one-way ANOVA with multiple comparisons corrected with Holm Sidak's test, or a t-test followed by Mann-Whitney $\mathrm{U}$ test on ranks and Wilcoxon test, with Bonferroni correction for multiple measurements. Correlation between tumor and tracer volumes was tested using Pearson correlation and linear regression. Significance levels were set at $p<0.05$. All results are shown as mean \pm SE or mean \pm SD. Only animals with full dataset were considered. Outliers were automatically excluded. 


\section{RESULTS}

\section{F-DPA-714 PET Imaging Reveals the Presence of Therapy-Resistant Cells in Chronically Treated Mice}

Both NT and preconditioned groups showed similar Gd-CE T1w-MRI and 18F-FET-based volume dynamics, together with 18F-DPA-714-based volume, indicating increased tumor volume (TV) and inflammation over time, as well as the NT group (Figure 1B and Figure 2). TV was significantly reduced in the chronically-treated group compared to the NT at day 14. Interestingly, 18F-DPA-714 signal was distributed at the borders and in the caudal parts of the tumors, especially in the chronically treated animals, highlighting spatial complementarities to 18F-FET uptake (Figure 1B).

The analyses of the tumor-to-background ratio displayed a significant reduction of 18F-DPA-714 uptake already at d7 in the chronically-treated group compared to the NT group (Supplemental Figure 2). See supplementary file for additional data (Supplemental Table 3\&4).

A high density of $\mathrm{Iba} 1^{+}$cells was detected in preconditioned and repopulated mice, as well as in the NT group. Chronic treatment reduced the number of $\mathrm{Iba}_{-1} \mathrm{1}^{+}$cells, although not as expected, revealing a resistant Iba $1^{+}$cell population in both hemispheres. Repopulation significantly increased the number of Iba $1^{+}$cells after preconditioning (Supplemental Figure 3 ). Those persistent cells were present within and at the periphery of the glioma and showed reduced CSF-1R and TSPO expression compared to the preconditioned and repopulated group, few of them expressed both markers. Interestingly, we observed the influx of apparent round-shaped CSF-1 ${ }^{+} \mathrm{TSPO}^{+}$cells within the tumor area (Figure 3).

\section{Volumetric Analyses Show Significant Reduction of Tumor- And Tracer Uptake-Volumes After Acute Treatment with Subsequent Repopulation (RM)}

We then assessed the possible therapeutic effects of acute treatment and subsequent microglial repopulation. In NT animals, Gd-CE T1w-MRI, 18F-FET- and 18F-DPA-714-PET showed significant increasing in TV and both tracer volume over time. In the PLX5622-treated group, Gd-CE T1w-MRI and 18F-FETderived volumes significantly increased between d7 and d21 while 18F-DPA-714-derived volume significantly increased between d7 and d14 (Figure 4B and Figure 5). Therapy effect was observed at day 
21, where repopulated animal showed significantly reduced TV, 18F-FET and 18F-DPA-714-derived volumes compared to NT animals. See supplementary files for additional data (Supplemental Table 5).

In the NT group, 18F-FET Tmean/Bmean significantly increased between d7 and d21 while in the PLX5622+Repopulation group it increased between d14 and d21. Similarly, 18F-FET Tmax/Bmean was increased in the NT group but significantly reduced in repopulated animals at d21 (Supplemental Figure 4, Supplemental Table 6).

In depth analyses showed a statistically significant positive correlation between MRI-based TV and 18F-FET uptake already at d14 and also at d21 (Supplemental Figure 7).

\section{GAMM are Significantly Affected by CSF-1R Inhibition with Persistent Reduction of CSF-1R and TSPO Expression After Drug Withdrawal}

PLX5622 treatment successfully depleted the majority of $\mathrm{Iba} 1^{+}$cells in glioma-bearing mice compared to NT group ( $p \leq 0.05)$, both in the ipsi- and contralateral-hemisphere, in line with TSPO-PET. The remaining $\mathrm{Iba}^{+}$cells were located at the periphery of the glioma or within the tumor borders, displaying an amoeboid-like morphology indicative of an active state. After one week of repopulation, the number of $\mathrm{Iba}^{-1}{ }^{+}$cells was significantly increased in the contralateral-side of the repopulation group compared to the PLX5622 group ( $p \leq 0.05)$ (Supplemental Figure 5).

Similarly, CD68 expression was analyzed as a marker for glioma-associated microglia/macrophages and specifically for macrophage-mediated immune suppression. A large number of $\mathrm{CD} 8^{+}$cells was detected infiltrating the glioma tissue in NT animals. The number of $\mathrm{CD} 68^{+}$cells was significantly reduced with PLX5622 treatment and remained significantly reduced after repopulation as shown by quantification (Supplemental Figure 6).

CSF-1 $\mathrm{R}^{+}$tumor cells, Iba $1^{+}$microglial cells at the periphery and infiltrating the glioma and CSF$1 \mathrm{R}^{+} \mathrm{Iba} 1^{+}$cells within the tumor mass were detected in NT animals. PLX5622 treatment reduced CSF-1R expression, with few CSF- $1 \mathrm{R}^{+} \mathrm{Iba} 1^{+}$cells present at the border of the glioma. After repopulation, CSF-1R signal remained reduced and CSF-1 $\mathrm{R}^{+}$cells were visible at the periphery of the glioma (Figure 6). 
Similarly, $\mathrm{TSPO}^{+}$tumor cells and $\mathrm{Iba} 1^{+}$cells, as well as $\mathrm{TSPO}^{+} \mathrm{Iba} 1^{+}$cells, were observed surrounding and infiltrating the glioma tissue in the NT group. After CSF-1R inhibition, TSPO signal was mostly detectable at the border of the tumor mass, in line with the imaging results, together with few $\mathrm{Iba} 1^{+}$and $\mathrm{TSPO}^{+} \mathrm{Iba} 1^{+}$ cells. Following repopulation and in accordance with the imaging results, the reduction of TSPO signal remained stable and $\mathrm{TSPO}^{+}$cells were visible at the periphery of the glioma while cells being $\mathrm{Iba}^{+}{ }^{+} \mathrm{TSPO}^{-}$ and $\mathrm{Iba} 1^{+} \mathrm{TSPO}^{+}$were detectable within the glioma tissue (Figure 7). Interestingly, the depletion of Iba1 ${ }^{+}$ cells seemed to promote the infiltration of as yet uncharacterized CSF-1R ${ }^{+}$TSPO $^{+}$cells (Figure 6 and 7 green frame detailed images).

\section{CSF-1R Inhibition Shows Immune-Modulatory Effects on Glioma-Associated Myeloid-Derived Cells}

To further evaluate therapy-induced changes in the TME and to characterize the phenotype of infiltrating cells, multiparametric flow cytometry was performed (Supplemental Figure 8). PLX5622 treatment reduced the frequency of $\mathrm{CD}^{4} 5^{+}$tumor-infiltrating leucocytes (TILs) compared to the NT group (15.2\% vs $22.3 \%$, respectively), which slightly increased after repopulation (19.1\%). The same results were observed regarding GAMMs, confirming the histological results. Comparable amounts of total MDSCs were detected in the PLX5622-treated and NT group (CD11b ${ }^{+}$Gr1 ${ }^{+}: 5.1 \%$ vs $3.6 \%$ ), which significantly decreased after repopulation (CD11 $\left.\mathrm{b}^{+} \mathrm{Gr} 1^{+}: 0.78 \%\right)$. Moreover, PLX5622 treatment produced a difference in the MDSCs phenotype and affected the expression of different M2 activation markers, such as TSPO and MHC-II. See the Appendix for additional information. 


\section{DISCUSSION}

This study aimed to assess the suitability of a multi-tracer PET/MRI combination to investigate the effects of microglia-depleting immunotherapy in the TME in a syngeneic mouse glioma model. Using different treatment regimens, we demonstrate that the dual tracer combination of 18F-FET- and 18F-DPA714-PET in conjunction with MRI allows monitoring therapy-induced changes in glioma progression and GAMM dynamics in the TME. In contrast to 18F-FET-PET, 18F-DPA-714-PET may be suitable to detect immunotherapy-induced changes within the glioma microenvironment when it is used in conjunction with detailed IHC analysis. We demonstrate that CSF-1R inhibition affects both, glioma cells and specific gliomaassociated myeloid-derived cell populations. GAMM inhibition was sustained even after drug withdrawal. Histological analyses confirmed the efficacy of PLX5622 to deplete the majority of $\mathrm{Iba} 1^{+}$cells over a short period, while a longer treatment resulted in the presence of a therapy-resistant cell population. Interestingly, results indicated that microglia cells and GAMMs do not seem essential for glioma initiation. However, a short-term brain repopulation period after CSF-1R therapy is highly effective in slowing glioma progression and maintaining a reduced inflammatory response. Finally, TSPO-PET using 18F-DPA-714 gives complementary information to the imaging marker 18F-FET. Altogether, these findings provide a new insight into CSF-1R therapy resistance in glioma, and identify novel therapeutic glioma targets.

The role of the CSF-1R/CSF-1 axis has been studied in many cancer types since tumor cells secrete CSF-1 to attract CSF-1 $\mathrm{R}^{+}$myeloid-derived cells to the TME to sustain tumor progression. Therefore, the expression levels of CSF-1R and circulating ligand are regarded as biomarkers for tumor progression, treatment failure and poor prognosis (19). Different CSF-1R inhibitors have been tested in pre-clinical studies and clinical trials but failed to show substantial efficacy due to acquired resistance to CSF-1R therapy by the glioma microenvironment (20).

TSPO is reported to be upregulated by resident (microglia, astrocytes) and peripheral immune cells (monocytes, lymphocytes, neutrophils) during inflammatory conditions (21). In glioma tissue, TSPO is 
expressed by glioma cells, GAMM and endothelial cells (22). Our group recently described the suitability of TSPO-PET using 18F-DPA-714 to monitor treatment outcomes in a preclinical glioma model, by identifying areas of myeloid cells infiltration within the glioma microenvironment (17).

Our results show the efficacy of CSF-1R inhibition for the blockade of GAMM proliferation and that the combination of 18F-FET and 18F-DPA-714-PET can be used to monitor CSF-1R therapy-induced changes in the glioma microenvironment. CSF-1R inhibitor PLX5622 proves to have a disease-changing effect, reducing glioma progression and neuroinflammation, particularly when administered for a short period during established glioma growth.

We report data on ablation of microglia cells and GAMMs before and during glioma development showing that the inhibition of these cell populations before glioma initiation does not influence glioma formation nor the inflammatory response. Ex vivo characterization revealed the presence of potentially resistant $\mathrm{Iba} 1^{+}$cell population as possible major cellular sources of TSPO expression, particularly in the chronically treated animals. These findings were supported by in-depth histological analyses that showed a persistent reduction of TSPO and CSF-1R signals coming from the glioma tissue, whereas a high number of $\mathrm{TSPO}^{+}$and $\mathrm{CSF}-1 \mathrm{R}^{+}$cells were infiltrating the glioma microenvironment. The same holds true for the preconditioned and repopulated group characterized by the presence of a high number of GAMM and microglia cells positive for all the markers infiltrating the glioma tissue. These results might explain the increased 18F-DPA-714 uptake over time, highlighting the importance of TSPO-PET in detecting gliomaassociated cells infiltration.

Whether the observed resistant cell population is pro- or anti-tumorigenic has yet to be clarified. However, they partially expressed TSPO, supporting the efficacy of TSPO-PET in monitoring specific gliomaassociated inflammation. Furthermore, TSPO-PET has a great potential for the characterization and imaging of the glioma-associated immunosuppressive TME, as shown in the first in-patients study by Zinnhardt et al. (14). 
The importance of choosing the proper therapeutic window for GAMM modulation is crucial, and TSPO-PET may serve this purpose (23). In this regard, a short term (1 week) CSF-1R inhibition with subsequent brain repopulation during glioma progression led to a significant reduction of tumor volume as quantified by both CE-Gd-MRI and 18F-FET-PET. In accordance with the literature, CSF-1R inhibition significantly reduced the number of $\mathrm{Iba}^{+}$cells in the brain (24). However, tumor cells also express TSPO and the volumetric analysis showed increased 18F-DPA-714-PET volumes at day 14 at the same level as the NT group due to glioma progression which may hide possible immunological-induced changes. Interestingly, after seven days of brain repopulation, 18F-DPA-714-PET signal remained stable and a significant reduction in tracer volume was detected compared to the control group, suggesting long-lasting treatment effects. These findings were further confirmed by histology. Furthermore, we showed that CSF$1 \mathrm{R}$ inhibition significantly reduced the number of GAMM infiltrating the glioma tissue, identified as $\mathrm{CD} 68^{+}$ cells, and the inhibitory effect was detected also after brain repopulation. These results demonstrate that GAMM are susceptible to acute CSF-1R-mediated intervention even after treatment withdrawal. In line with the results obtained after the chronic treatment and as discussed above, fluorescent labeling revealed the presence of TSPO and CSF-1R positive immune cells infiltrating the tumor after depletion of microglia cells. The presence of these potentially therapy-resistant cells supports the importance of microglia in preventing the influx of tumor-associated cells within the TME. Accordingly, previous studies reported increased peripheral immune cell infiltration following microglia depletion $(25,26)$.

The CSF-1R therapy-induced effects on the immune component were further investigated by multiparametric flow cytometry analyses. Overall, the analyses indicated a strong effect of CSF-1R immune-modulation on the MDSCs population. The results suggest that the infiltrating component in the absence of Iba $1^{+}$cells might be represented by PMN-MDSCs. Moreover, PLX5622 effects resulted in the modulation of MHC class II and TSPO expression on GAMM and MDSCs, in line with the reduction of 18FDPA-714-PET volumes. This may indicate that repopulation could modulate their immunosuppressive function toward an anti-tumor phenotype, highlighting the importance of targeting GAMM. 
As reported, single-agent therapy with CSF-1R inhibitors has demonstrated only modest results in glioblastoma clinical trials, showing no significant improvement of the progression-free survival of the patients (27). Currently, other studies are ongoing combining CSF-1R therapy and immune-checkpoint inhibitors in different types of tumors (28). One pilot study evaluating the TSPO-PET tracer 11C-PBR28 in patients with primary GLIOBLASTOMA MULTIFORME or melanoma brain metastasis, treated with chemoradiation or immunotherapy, has been completed recently and results are to be determined (NCT02431572) (29).

The validation of TSPO-PET tracers in clinical settings is necessary to improve the understanding of glioma-associated inflammation and therapy resistance mechanisms.

\section{CONCLUSION}

In conclusion, 18F-DPA-714 may be a useful imaging biomarker for longitudinal therapy monitoring and assessment of the glioma-associated inflammatory microenvironment, as well as for patient stratification. CSF-1R-targeting intervention and determination of the ideal treatment window for CSF-1R inhibitors may define a promising complementary therapy strategy in glioma.

\section{ACKNOWLEDGMENTS}

The authors thank Sarah Köster, Christine Bätza, Roman Priebe, Stefanie Bouma, Irmgard Hoppe, Christa Möllmann, Nina Kreienkamp and Dirk Reinhardt for their excellent technical support, as well as Christian Döring for support on the volumetric analyses.

\section{DISCLOSURE}

The authors declare no conflict of interests. 


\section{KEY POINTS}

Question: Is 18F-DPA-714-PET suitable to decipher glioma-associated immune cell dynamics and CSF-1R therapy outcomes?

Pertinent Findings: We show that 18F-DPA-714-PET provide complementary information to 18F-FET-PET on glioma heterogeneity, allows the imaging of immune cell dynamics, supports the assessment of brain target engagement and elucidates CSF-1R inhibition-induced effects in vivo.

Implications for Patient Care: 18F-DPA-714-PET represents a promising tool for non-invasively imaging of therapeutic modulations of the immunosuppressive glioma microenvironment. 


\section{REFERENCES}

1. Stupp R, Mason WP, van den Bent MJ, et al. Radiotherapy plus concomitant and adjuvant temozolomide for glioblastoma. N Engl J Med. 2005;352:987-996.

2. Xu S, Tang L, Li X, Fan F, Liu Z. Immunotherapy for glioma: current management and future application. Cancer Lett. 2020;476:1-12.

3. DeCordova S, Shastri A, Tsolaki AG, et al. Molecular heterogeneity and immunosuppressive microenvironment in glioblastoma. Front Immunol. 2020;11:1402.

4. Shabab T, Khanabdali R, Moghadamtousi SZ, Kadir HA, Mohan G. Neuroinflammation pathways: a general review. Int J Neurosci. 2017;127:624-633.

5. Rojo R, Raper A, Ozdemir DD, et al. Deletion of a Csf1r enhancer selectively impacts CSF1R expression and development of tissue macrophage populations. Nat Commun. 2019;10:3215.

6. Albert NL, Weller M, Suchorska B, et al. Response assessment in neuro-oncology working group and european association for neuro-oncology recommendations for the clinical use of PET imaging in gliomas. Neuro Oncol. 2016;18:1199-1208.

7. Lundemann M, Munck af Rosenschöld P, Muhic A, et al. Feasibility of multi-parametric PET and MRI for prediction of tumour recurrence in patients with glioblastoma. Eur J Nucl Med Mol Imaging. 2019;46:603-613.

8. Stegmayr C, Willuweit A, Lohmann P, Langen K-J. O-(2-[18F]-Fluoroethyl)-L-Tyrosine (FET) in neurooncology: a review of experimental results. Curr Radiopharm. 2019;12:201-210.

9. Dhermain FG, Hau P, Lanfermann $\mathrm{H}$, Jacobs AH, van den Bent MJ. Advanced MRI and PET imaging for assessment of treatment response in patients with gliomas. Lancet Neurol. 2010;9:906-920.

10. Moreau A, Febvey O, Mognetti T, Frappaz D, Kryza D. Contribution of different positron emission tomography tracers in glioma management: focus on glioblastoma. Front Oncol. 2019;9:1134.

11. Cai L, Kirchleitner S V, Zhao D, et al. Glioblastoma exhibits inter-individual heterogeneity of TSPO and LAT1 expression in neoplastic and parenchymal cells. Int J Mol Sci. 2020;21:612.

12. Dupont A-C, Largeau B, Santiago Ribeiro M, Guilloteau D, Tronel C, Arlicot N. Translocator Protein-18 kDa (TSPO) Positron Emission Tomography (PET) imaging and its clinical impact in neurodegenerative diseases. Int J Mol Sci. 2017;18:785.

13. Zinnhardt B, Pigeon $\mathrm{H}$, Thézé B, et al. Combined PET imaging of the inflammatory tumor microenvironment identifies margins of unique radiotracer uptake. Cancer Res. 2017;77:1831-1841.

14. Zinnhardt B, Müther M, Roll W, et al. TSPO imaging-guided characterization of the 
immunosuppressive myeloid tumor microenvironment in patients with malignant glioma. Neuro Oncol. 2020;22:1030-1043.

15. Foray C, Barca C, Backhaus $\mathrm{P}$, et al. Multimodal molecular imaging of the tumour microenvironment. In: Advances in Experimental Medicine and Biology. Vol 1225. NLM (Medline); 2020:71-87.

16. Percie du Sert N, Ahluwalia A, Alam S, et al. Reporting animal research: Explanation and elaboration for the ARRIVE guidelines 2.0. Boutron I, ed. PLOS Biol. 2020;18:e3000411.

17. Foray C, Valtorta S, Barca C, et al. Imaging temozolomide-induced changes in the myeloid glioma microenvironment. Theranostics. 2021;11:2020-2033.

18. Bankhead P, Loughrey MB, Fernández JA, et al. QuPath: Open source software for digital pathology image analysis. Sci Rep. 2017;7:16878.

19. Achkova D, Maher J. Role of the colony-stimulating factor (CSF)/CSF-1 receptor axis in cancer. Biochem Soc Trans. 2016;44:333-341.

20. Quail DF, Joyce JA. Molecular pathways: deciphering mechanisms of resistance to macrophage-targeted therapies. Clin Cancer Res. 2017;23:876-884.

21. Fujinaga M, Luo R, Kumata K, et al. Development of a 18 F-labeled radiotracer with improved brain kinetics for positron emission tomography imaging of translocator protein (18 kDa) in Ischemic Brain and Glioma. J Med Chem. 2017;60:4047-4061.

22. Winkeler A, Boisgard R, Awde AR, et al. The translocator protein ligand [18F]DPA-714 images glioma and activated microglia in vivo. Eur J Nucl Med Mol Imaging. 2012;39:811823.

23. Press RH, Zhong J, Gurbani SS, et al. The role of standard and advanced imaging for the management of brain malignancies from a radiation oncology standpoint. Neurosurgery. 2019;85:165-179.

24. Elmore MRP, Najafi AR, Koike MA, et al. Colony-stimulating factor 1 receptor signaling is necessary for microglia viability, unmasking a microglia progenitor cell in the adult brain. Neuron. 2014;82:380-397.

25. Otxoa-de-Amezaga A, Miró-Mur F, Pedragosa J, et al. Microglial cell loss after ischemic stroke favors brain neutrophil accumulation. Acta Neuropathol. 2019;137:321-341.

26. Unger MS, Schernthaner $\mathrm{P}$, Marschallinger J, Mrowetz $\mathrm{H}$, Aigner L. Microglia prevent peripheral immune cell invasion and promote an anti-inflammatory environment in the brain of APP-PS1 transgenic mice. J Neuroinflammation. 2018;15:274.

27. Lamb YN. Pexidartinib: First Approval. Drugs. 2019;79:1805-1812.

28. Benner B, Good L, Quiroga D, et al. Pexidartinib, a novel small molecule CSF-1R inhibitor in use for tenosynovial giant cell tumor: a systematic review of pre-clinical and clinical development. Drug Des Devel Ther. 2020;Vol 14:1693-1704. 
29. Gerstner ER. A pilot study to evaluate PBR PET in brain tumor patients treated with chemoradiation or immunotherapy - Full Text View - ClinicalTrials.gov.

https://clinicaltrials.gov/ct2/show/NCT02431572?term=tspo\&cond=Glioma\&draw=2\&ran $k=1$. 
A

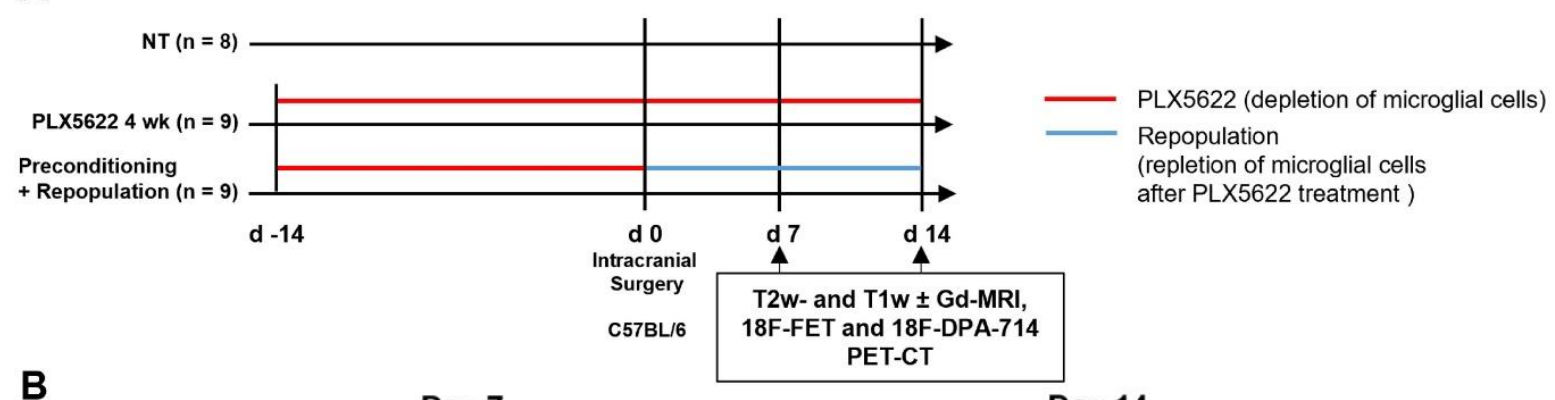

B

Day 7

Day 14
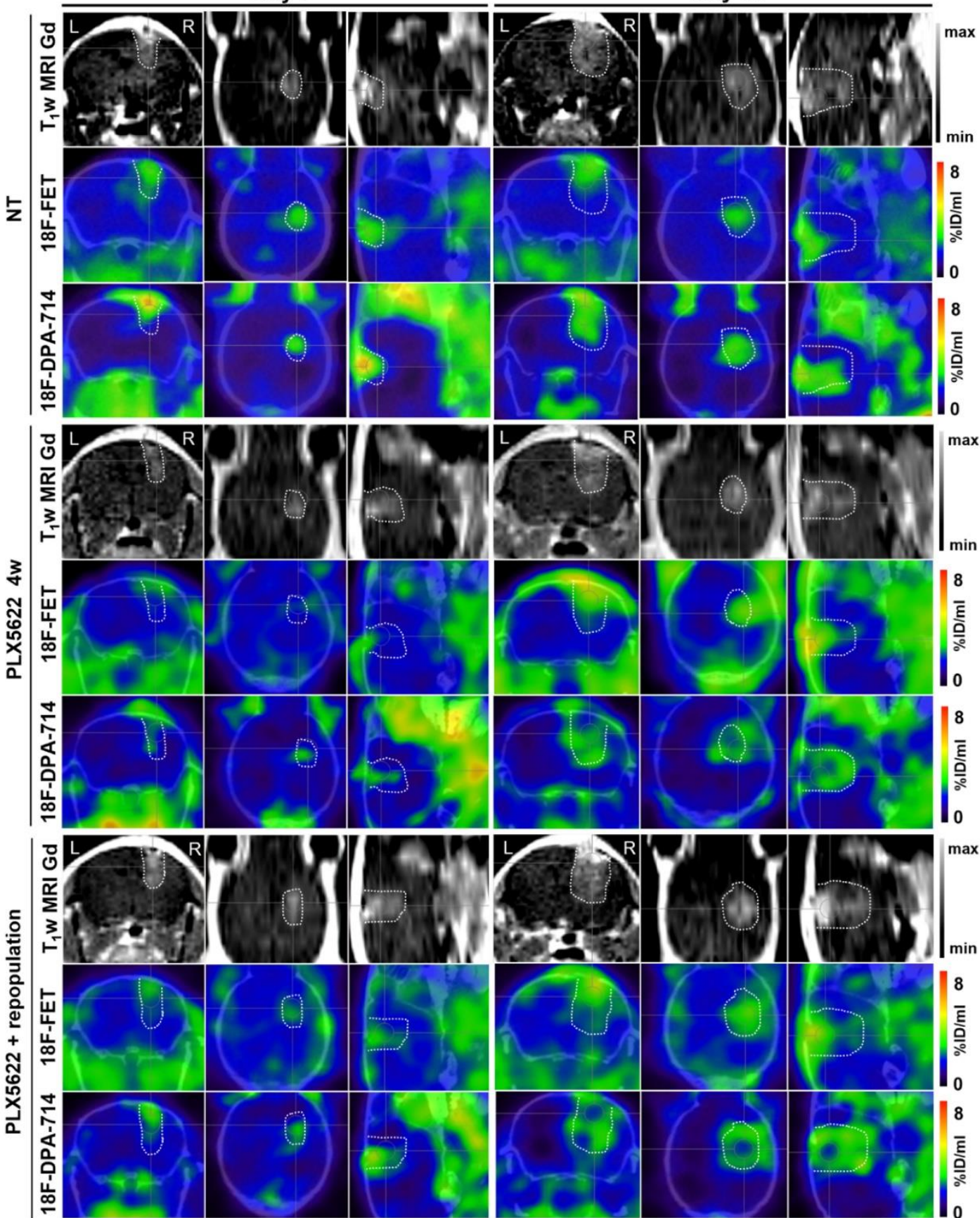
Figure 1. Suitability of longitudinal Gd-CE-T1w-MR, 18F-FET- and 18F-DPA-714-PET imaging in monitoring immunotherapy-induced changes - preconditioning model (PM). (A) Workflow of the preconditioning model. (B) CE-T1w-MR and PET images for 18F-FET and 18F-DPA-714 (top to bottom) during different PLX5622 regimens (left to right). Dotted line: tumor area depicted by MRI and transferred to PET images. 4 wk: 4 weeks; CE-MR: contrast-enhanced MR; L and R: left- and right-hemisphere. 

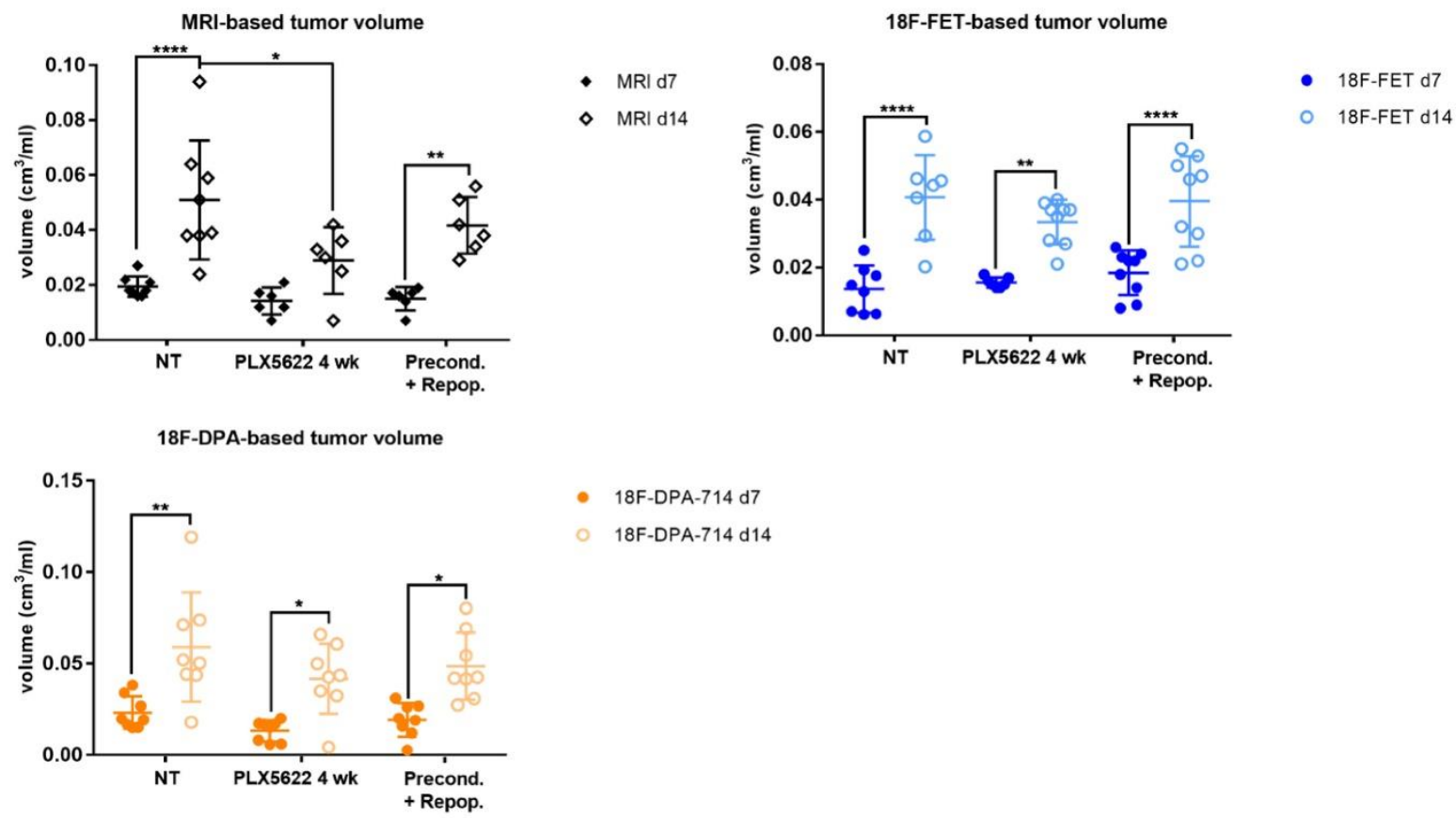

Figure 2. Analysis of tumor and tracer volumes of the preconditioning model. Volumetric analysis of CET1w-MR-, (C) 18F-FET- and (D) 18F-DPA-714-derived TV, in the experimental groups. $\mathrm{N}=8 \mathrm{NT} ; \mathrm{n}=9$ PLX5622 4 wk; $n=9$ Precond. + Repop. ${ }^{*} p \leq 0.05 ;{ }^{* *} p \leq 0.01 ;{ }^{* *} p \leq 0.001 ;{ }^{* * * *} p \leq 0.0001 .4$ w: 4 weeks; CE-MR: contrast-enhanced MR; NT: non-treated; TV: tumor volume. 

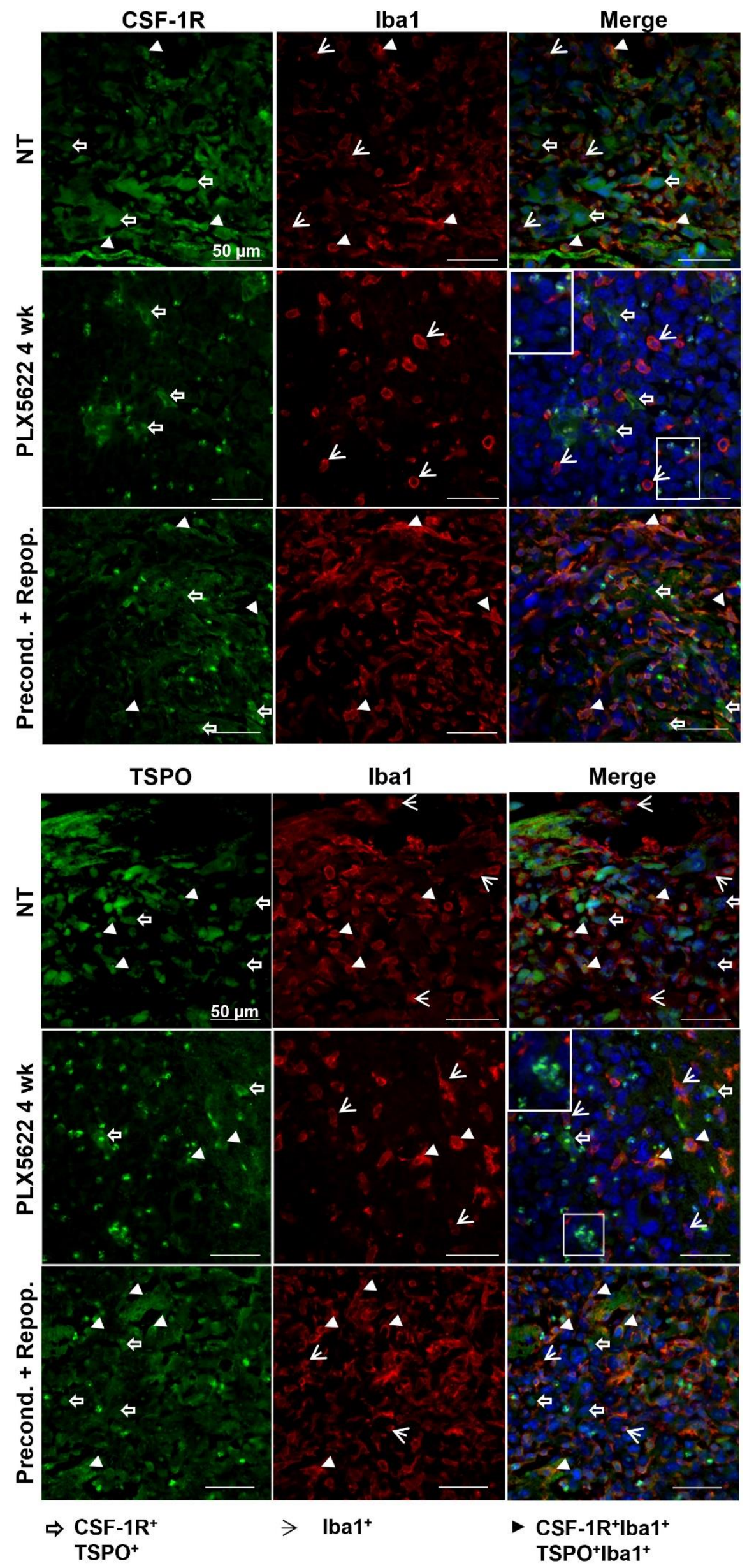
Figure 3. Dual-labeling for CSF-1R/Iba1 and TSPO/Iba1 confirms the presence of therapy-resistant cell populations. Paraffin embedded tissues labeled for CSF-1R/TSPO/Iba1. White frame image: unclassified infiltrating cells. In blue the nuclear staining with DAPI. ( $N=3$ /group). CSF-1R: colony stimulating factor-1 receptor; TSPO: translocator protein. 
A

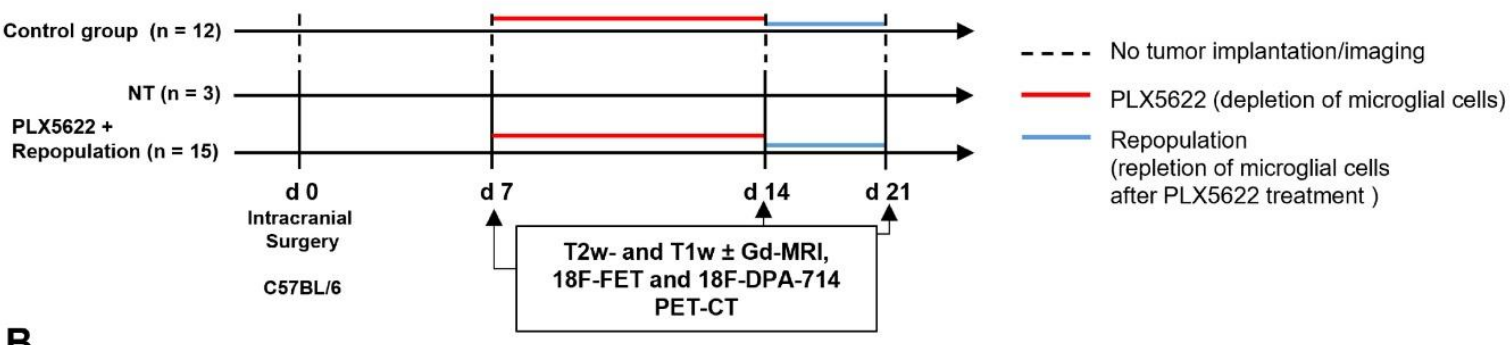

B

Day 7

Day 14

Day 21

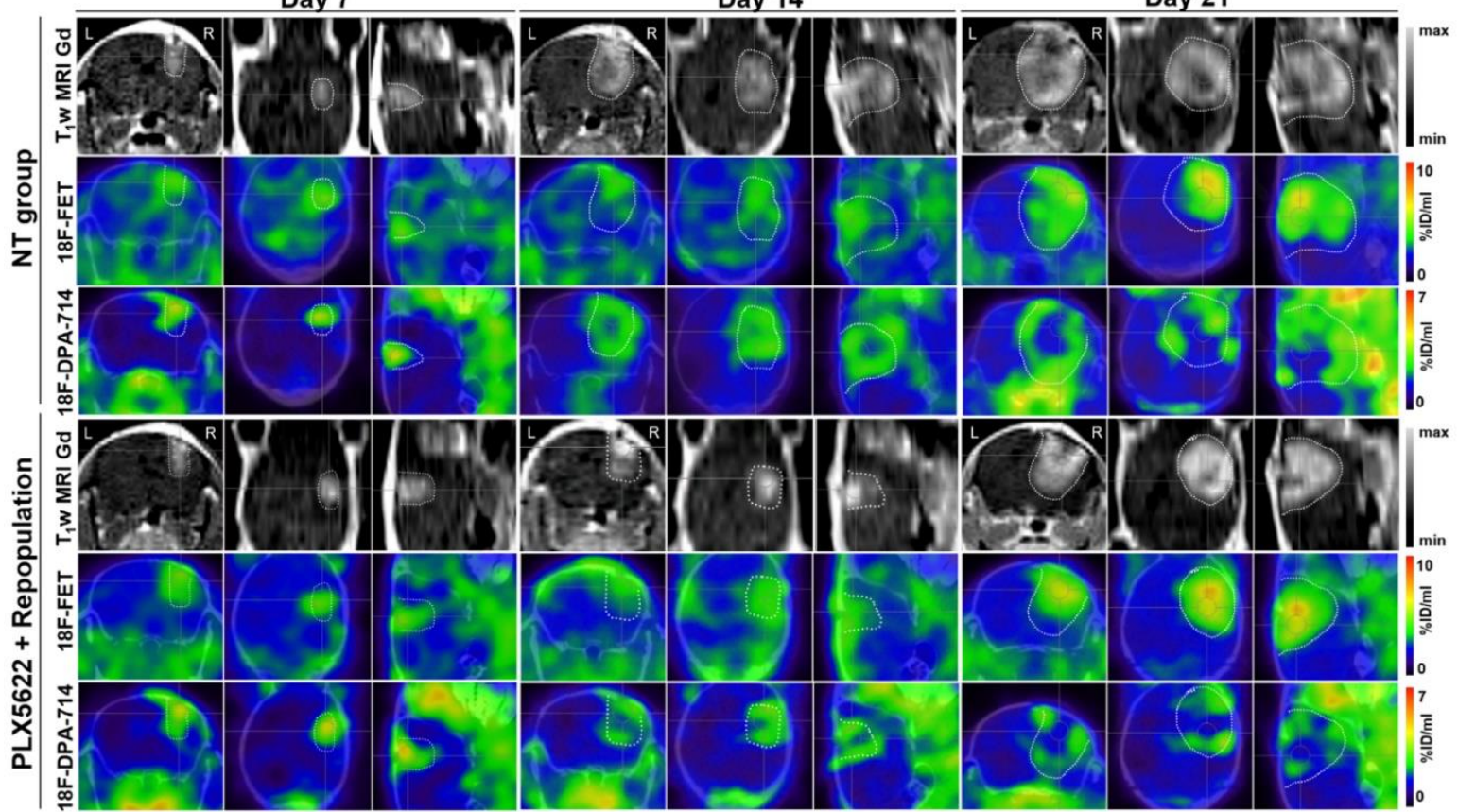

Figure 4. Monitoring glioma immunotherapy-induced changes after GAMM depletion and repopulation using multimodal PET/MRI. (A) Workflow of the repopulation model. (B) CE-T1w-MR and PET images for 18F-FET and 18F-DPA-714 (top to bottom) of NT and PLX5622-treated animals, pre-, post-treatment, and after GAMMs repopulation (left to right). Dotted line: tumor area depicted by MRI and transferred to PET images. CE-MR: contrast-enhanced MR; GAMMs: glioma-associated microglia/macrophages; L and R: leftand right-hemisphere; NT: non-treated. 

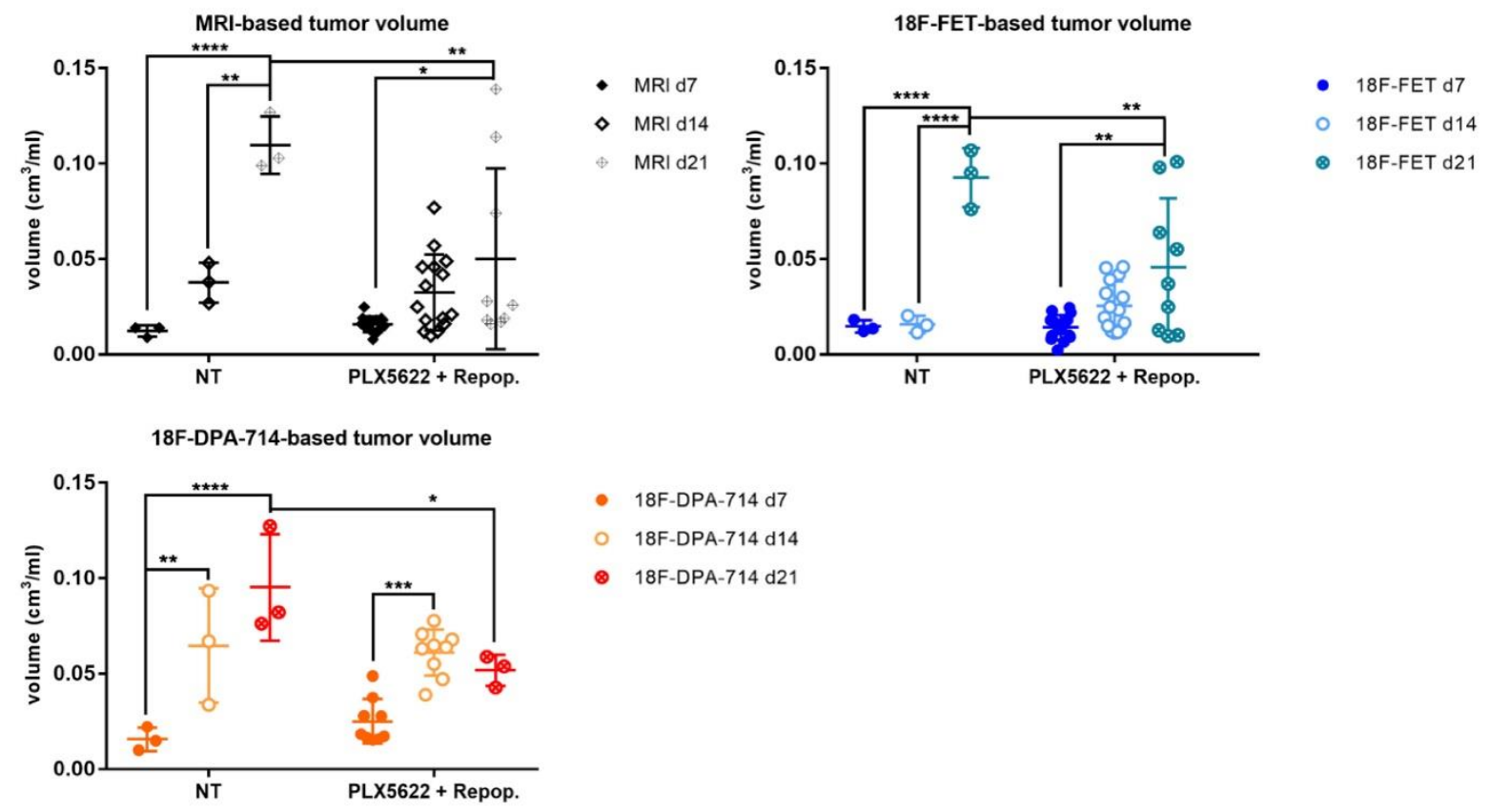

Figure 5. Analysis of tumor and tracer volumes of the repopulation model. Volumetric analysis of CE-T1wMR-, 18F-FET- and 18F-DPA-714-derived TV, in NT and PLX5622-treated and repopulated-group. N = 3 NT; $\mathrm{n}=15$ PLX5622+repop. ${ }^{*} \mathrm{p} \leq 0.05 ;{ }^{* *} \mathrm{p} \leq 0.01 ;{ }^{* *} \mathrm{p} \leq 0.001$. CE-MR: contrast-enhanced MR; NT: nontreated; TV: tumor volume. 


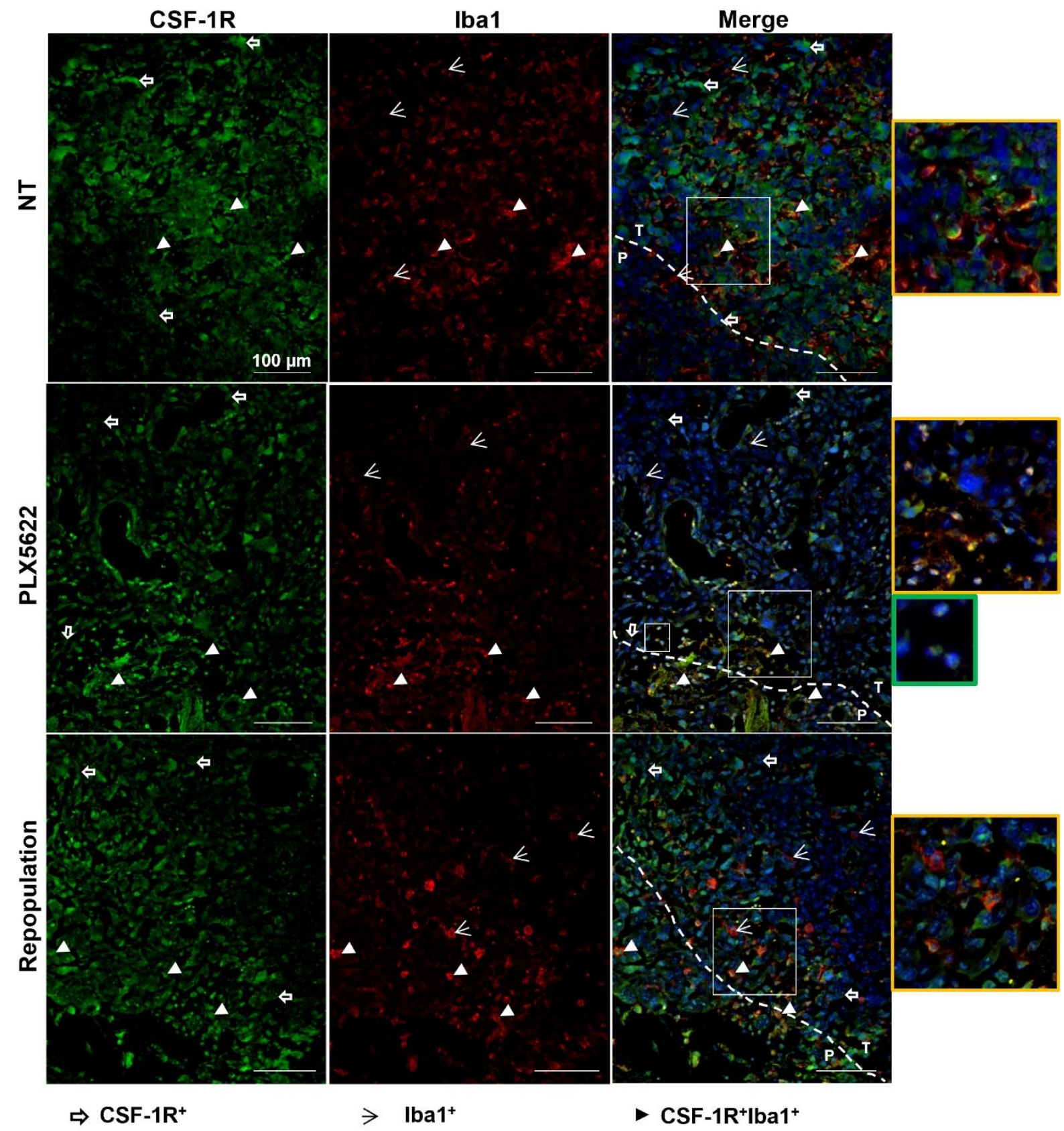

Figure 6. Immunofluorescence labeling for CSF-1R and Iba1 shows persistent reduction of CSF-1R signal after repopulation. Paraffin embedded tissue labeled for CSF-1R (green) and Iba1 (red) in NT, PLX5622treated and repopulated group. In blue the nuclear staining with DAPI. Yellow frame images: magnified details. Green frame image: unclassified infiltrating cells. Dotted white line: separation between periphery and inner mass. ( $n=3 /$ group). CSF-1R: colony stimulating factor-1 receptor; NT: non-treated; P: periphery; T: tumor. 


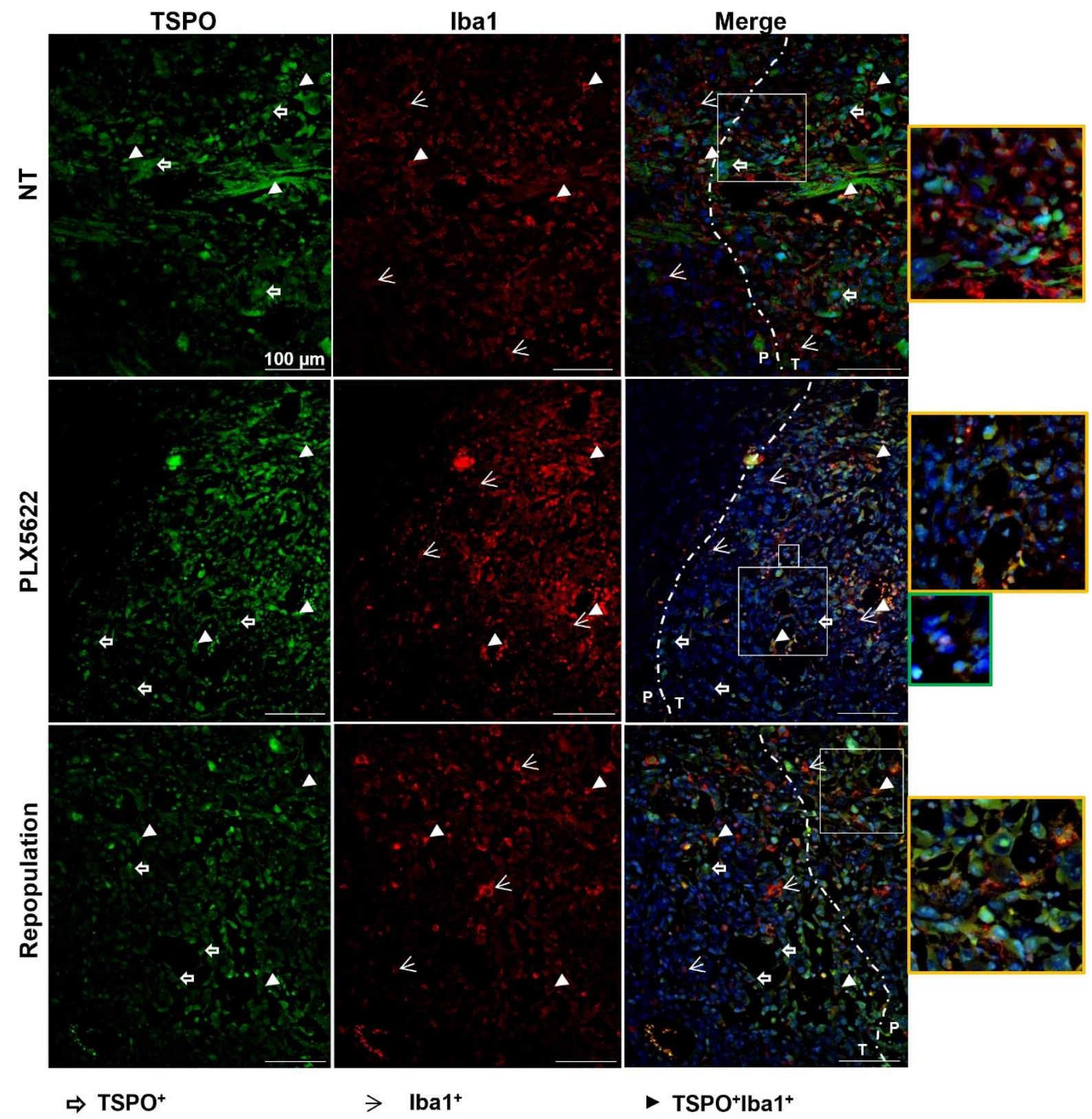

Figure 7. Immunofluorescence labeling for TSPO and Iba1 shows persistent reduction of TSPO signal after repopulation. Paraffin embedded tissue labeled for TSPO (green) and Iba1 (red) in NT, PLX5622-treated and repopulated group. In blue the nuclear staining with DAPI. Yellow frame images: magnified details. Green frame image: unclassified infiltrating cells. Dotted white line: separation between periphery and inner mass. ( $\mathrm{N}=3$ /group). TSPO: translocator protein; NT: non-treated; P: periphery; T: tumor. 
Graphical abstract

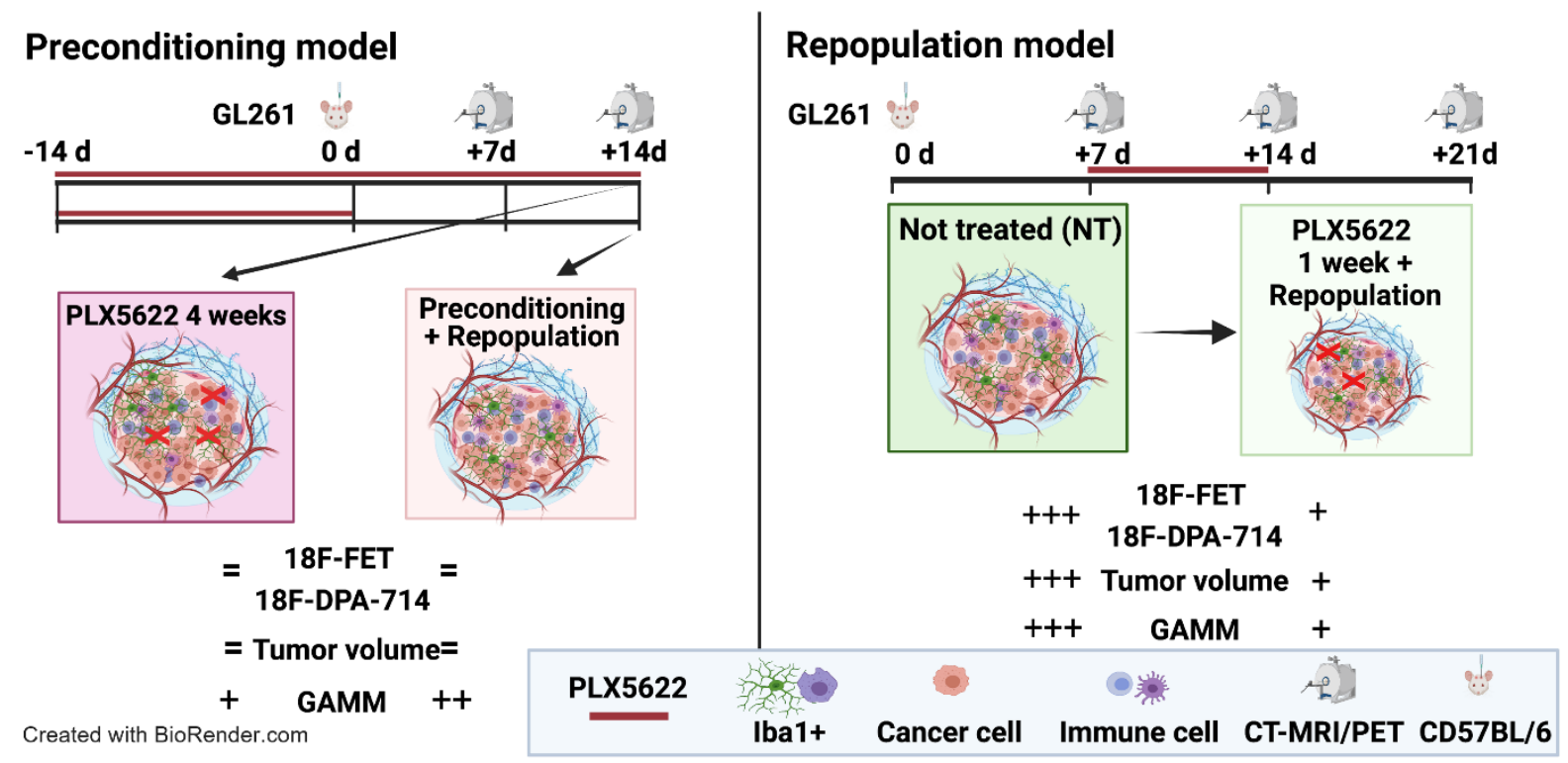




\section{Supplementary M\&M}

\section{Study design}

\section{- Preconditioning model (PM)}

$\mathrm{N}=26$ mice underwent the imaging protocol of sequential T1w-MRI, with and without gadolinium (Gd), and $\left[{ }^{18} \mathrm{~F}\right] \mathrm{FET}$ - and $\left[{ }^{18} \mathrm{~F}\right] \mathrm{DPA}-714-\mathrm{PET}-\mathrm{CT}$ as described previously (17). First, T1w-MRI (-/+Gd) was performed at day 7 post implantation (p.i.), followed by $\left[{ }^{18} \mathrm{~F}\right] \mathrm{FET}-\mathrm{PET}$ on the same day, and $\left[{ }^{18} \mathrm{~F}\right] \mathrm{DPA}-714-\mathrm{PET}$ on the next day. The same acquisitions were performed again 14 (T2w-MRI, $\left.\left[{ }^{18} \mathrm{~F}\right] \mathrm{FET}-\mathrm{PET}-\mathrm{CT}\right)$ and 15 days ([$\left.{ }^{18} \mathrm{~F}\right] \mathrm{DPA}-714$ PET-CT) p.i. (Figure 1A).

$\mathrm{N}=8$ mice constituted the control group while $\mathrm{n}=18$ mice were fed with special chow containing PLX5622 (1200 ppm; Plexxikon) starting 14 days prior to surgery. After surgery, the animals were divided randomly in three groups: the control group (NT; $n=8$ ), the chronically treated group fed with CSF-1R inhibitor until day 14 p.i. (PLX5622 4 wk; $n=9$ ), and the preconditioned and repopulated group that was fed with a control diet starting after surgery until day 14 p.i. (Preconditioning + Repopulation; $n=9$ ). The mice were sacrificed after the last imaging scan at day 15 p.i. and the ex-vivo analysis were immediately performed.

\section{- Acute treatment and repopulation model (RM)}

A total of $n=31$ C57BL/6 mice were employed during the repopulation study and 18 of them were successfully imaged at all the different time points, pre- ( 7 days p.i.) and post-therapy (14 days p.i.), and 7 days after drug withdrawal allowing brain repopulation (21 days p.i.). Two mice died during surgery and three mice died during the imaging sessions invalidating also the imaging data already acquired that were not included in the analysis. The same protocol of sequential imaging as described above was used (Figure 4A). After surgery, mice were divided into two main groups: a control group (NT; $n=11$ in total, 3 were included in the analysis) fed with control diet until day 21, and a PLX5622-treated and repopulated group (PLX5622 + Repopulation; $n=15)$. The latter group is composed by: a PLX5622-treated group (1 week of treatment starting at day 7 p.i., mice were sacrificed at day 15 p.i. after the last imaging scan; $n=11$ in total, 6 were included in the analysis) and a repopulation group 
( $n=9,6$ were excluded only in the 18F-DPA-714 volume analysis due to technical problems during the radiotracer synthesis at d21) that, after one week of PLX5622 treatment, was fed with control diet for 7 days (repopulation phase) and sacrificed at day 21 p.i. The ex-vivo analysis begun after the last imaging scan at day 21 p.i.

$\mathrm{N}=12$ mice did not undergo surgery and imaging sessions but were treated with CSF-1R inhibitor for one week. Six of them underwent brain repopulation and were sacrificed one week after drug withdrawal, constituting the control group to assess the efficacy of the drug under physiological conditions.

\section{PET studies protocol}

PET data were 3D reconstructed using one-pass list mode expectation maximization algorithm with resolution recovery. After each PET acquisition, the animal bed was transferred into the computed tomography (CT) scanner (Inveon, Siemens Medical Solutions) for anatomic co-registration using a landmark-based approach.

\section{Multiparametric flow cytometry gating strategy}

$\mathrm{CD} 45^{+}$cells were selected in a side scatter (SSC) vs CD45 plot. Viable cells were selected in a 7-AAD vs CD45 plot and then other subsets were defined: $\mathrm{CD} 11 \mathrm{~b}^{+} \mathrm{Gr} 1^{+}$(MDSCs), Ly6G+Ly6C- (PMN-MDSCs), Ly6G-Ly6C (Mo-MDSCs),

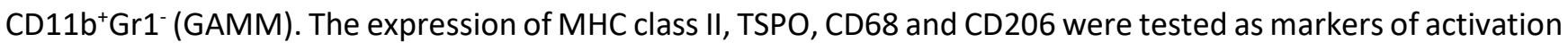
and M2-like phenotype. 
Supplemental Table 1. List of IHC/IF antibodies

\begin{tabular}{|l|c|c|c|}
\hline \multicolumn{1}{|c|}{ Primary Antibody } & Dilution & Species & Batch and Producer \\
\hline Iba1 & $1: 250$ & rabbit & 019-19741, FUJUFILM Wako Chemicals, Neuss, Germany \\
\hline TSPO & $1: 250$ & rabbit & ab109497, Abcam, Cambridge UK \\
\hline GFAP & $1: 500$ & chicken & ab13970, Abcam, Cambridge UK \\
\hline CSF-1R & $1: 100$ & rabbit & SAB1302603, Merck, Darmstadt, Germany \\
\hline CD68 & $1: 250$ & rabbit & ab125212, Abcam, Cambridge UK \\
\hline Secondary Antibody & Dilution & Species & Batch and Producer \\
\hline Alexa Fluor 488 & $1: 1000$ & rabbit & A-21206, life technologies, Carlsbad, USA \\
\hline Alexa Fluor 555 & $1: 1000$ & rabbit & A-21432, life technologies, Carlsbad, USA \\
\hline Iba1(635)-conjugated & $1: 200$ & rabbit & 013-26471, FUJUFILM Wako Chemicals, Neuss, Germany \\
\hline
\end{tabular}




\section{Supplemental Table 2. List of FACS antibodies}

\begin{tabular}{|l|l|}
\hline Antibody & Producer \\
\hline 7-AAD & \\
PerCP/Cy5.5 anti-Ly6G & \\
APC anti-CD11b & BioLegend, San Diego, California, USA \\
AlexaFluor 700 anti-CD45 & \\
APC/Fire 750 anti-Ly6C & \\
Brilliant Violet 421 anti-CD68 & \\
Brilliant Violet 510 anti-IA/IE & \\
FITC Isotope control antibody & \\
\hline AlexaFluor 488 recombinant anti-PBR (EPR5384) & Abcam, Cambridge, UK \\
\hline
\end{tabular}




\section{Supplemental data}

Supplemental Table 3. Statistical results of the volumetric analysis of the preconditioning study

\begin{tabular}{|c|c|c|c|c|c|c|}
\hline & \multicolumn{6}{|c|}{ Gd-CE T1wMRI (d14 vs d7) } \\
\hline & Mean 1 & Mean 2 & Mean Diff. & SE of diff. & Adjusted P Value & Summary \\
\hline NT & 0.05 & 0.019 & 0.031 & 0.006 & $<0.0001$ & **** \\
\hline PLX5622 4 wk & 0.029 & 0.014 & 0.015 & 0.007 & 0.31 & ns \\
\hline $\begin{array}{l}\text { Precondition + } \\
\text { Repopulation }\end{array}$ & 0.042 & 0.015 & 0.027 & 0.007 & 0.004 & ** \\
\hline \multirow[t]{3}{*}{$\begin{array}{c}\text { NT d14 } \\
\text { vs } \\
\text { PLX5622 } 4 \text { wk d14 }\end{array}$} & 0.05 & 0.03 & 0.02 & 0.006 & 0.015 & * \\
\hline & \multicolumn{6}{|c|}{ 18F-FET-PET (d14 vs d7) } \\
\hline & Mean 1 & Mean 2 & Mean Diff. & SE of diff. & Adjusted P Value & Summary \\
\hline NT & 0.041 & 0.014 & 0.027 & 0.005 & $<0.0001$ & **** \\
\hline PLX5622 4 wk & 0.033 & 0.016 & 0.017 & 0.004 & 0.002 & ** \\
\hline \multirow[t]{3}{*}{$\begin{array}{l}\text { Precondition + } \\
\text { Repopulation }\end{array}$} & 0.040 & 0.018 & 0.022 & 0.004 & $<0.0001$ & **** \\
\hline & \multicolumn{6}{|c|}{ 18-DPA-714 PET (d14 vs d7) } \\
\hline & Mean 1 & Mean 2 & Mean Diff. & SE of diff. & Adjusted P Value & Summary \\
\hline NT & 0.06 & 0.023 & 0.037 & 0.009 & 0.0014 & ** \\
\hline PLX5622 4 wk & 0.042 & 0.013 & 0.029 & 0.009 & 0.017 & * \\
\hline $\begin{array}{l}\text { Precondition + } \\
\text { Repopulation }\end{array}$ & 0.049 & 0.019 & 0.030 & 0.009 & 0.013 & * \\
\hline
\end{tabular}

Abbreviation: 4w: 4 weeks; Gd-CE: Gadolinium contrast enhanced. All data are volumes in $\mathrm{cm}^{3}$. 
Supplemental Table 4. Statistical results of the T/B ratios of the preconditioning study

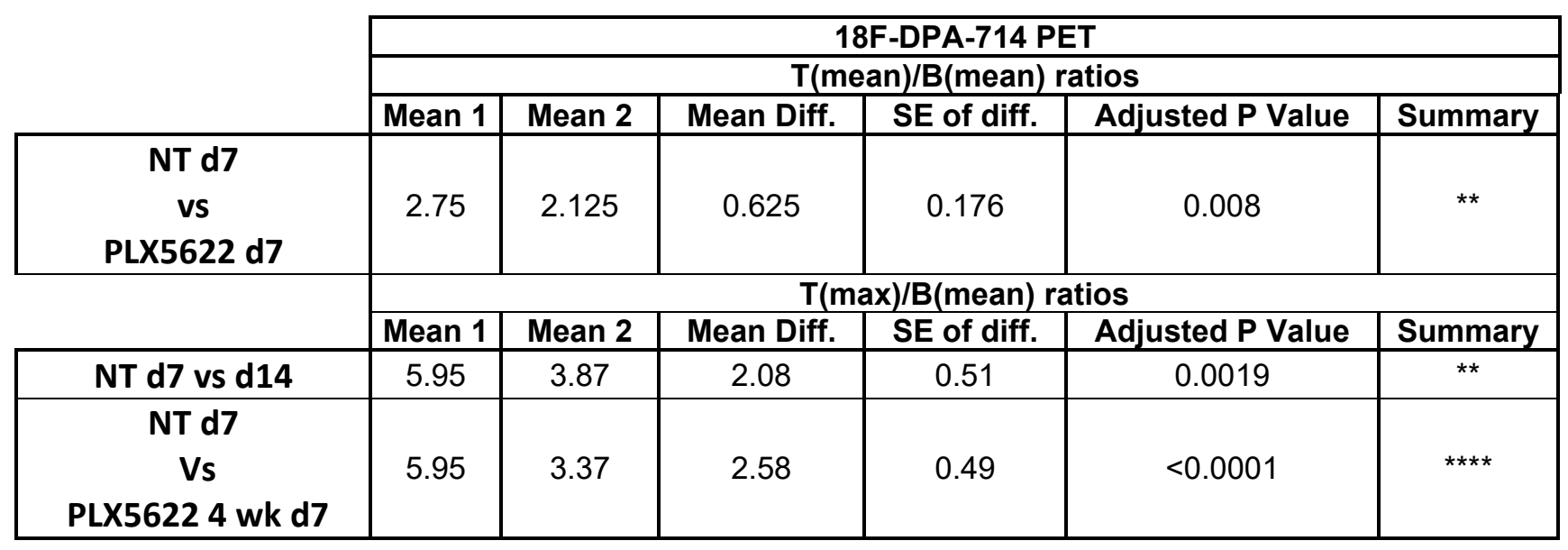


Supplemental Table 5. Statistical results of the volumetric analysis of the repopulation study

\begin{tabular}{|c|c|c|c|c|c|c|c|}
\hline & \multicolumn{7}{|c|}{ Gd-CE T1wMRI } \\
\hline & \multicolumn{3}{|c|}{ NT group } & \multicolumn{3}{|c|}{ PLX5622 + Repopulation } & \multirow{2}{*}{$\begin{array}{c}\text { NT vs } \\
\text { Repopulation } \\
\text { d21 vs d21 } \\
\end{array}$} \\
\hline & d14 vs d7 & d21 vs d7 & d21 vs d14 & d14 vs d7 & d21 vs d7 & d21 vs d14 & \\
\hline Mean 1 & 0.038 & 0.11 & 0.11 & 0.032 & 0.05 & 0.05 & 0.11 \\
\hline Mean 2 & 0.012 & 0.012 & 0.038 & 0.016 & 0.016 & 0.032 & 0.05 \\
\hline Mean Diff. & 0.025 & 0.097 & 0.072 & 0.016 & 0.034 & 0.018 & 0.06 \\
\hline SE of diff. & 0.02 & 0.02 & 0.02 & 0.008 & 0.01 & 0.01 & 0.016 \\
\hline Adjusted P Value & 0.83 & $<0.0001$ & 0.0055 & 0.41 & 0.012 & 0.52 & 0.0048 \\
\hline Significance & $\mathrm{ns}$ & $* * * *$ & $* *$ & ns & $*$ & ns & $* *$ \\
\hline \multicolumn{8}{|c|}{ 18F-FET-PET } \\
\hline Mean 1 & 0.016 & 0.093 & 0.093 & 0.025 & 0.046 & 0.046 & 0.093 \\
\hline Mean 2 & 0.015 & 0.015 & 0.016 & 0.014 & 0.014 & 0.025 & 0.046 \\
\hline Mean Diff. & 0.001 & 0.078 & 0.077 & 0.011 & 0.032 & 0.021 & 0.047 \\
\hline SE of diff. & 0.015 & 0.015 & 0.015 & 0.007 & 0.007 & 0.007 & 0.012 \\
\hline Adjusted P Value & $>0.9999$ & $<0.0001$ & $<0.0001$ & 0.54 & 0.0013 & 0.08 & 0.0029 \\
\hline Significance & ns & $* * * *$ & $* * * *$ & ns & $* *$ & ns & $* *$ \\
\hline \multicolumn{8}{|c|}{ 18F-DPA-714 PET } \\
\hline Mean 1 & 0.061 & 0.052 & 0.052 & 0.065 & 0.095 & 0.095 & 0.095 \\
\hline Mean 2 & 0.025 & 0.025 & 0.061 & 0.016 & 0.016 & 0.065 & 0.052 \\
\hline Mean Diff. & 0.036 & 0.027 & 0.0093 & 0.049 & 0.08 & 0.03 & 0.043 \\
\hline SE of diff. & 0.007 & 0.01 & 0.01 & 0.001 & 0.013 & 0.013 & 0.013 \\
\hline Adjusted P Value & 0.0004 & 0.11 & 0.96 & 0.0052 & $<0.0001$ & 0.16 & 0.015 \\
\hline Significance & $* * *$ & ns & $\mathrm{ns}$ & $* *$ & $* * * *$ & $\mathrm{~ns}$ & * \\
\hline
\end{tabular}

Abbreviation: NT: non treated; Gd-CE: Gadolinium contrast enhanced. All data are volumes in $\mathrm{cm}^{3}$ 
Supplemental Table 6. Statistical results of the T/B ratios of the repopulation study

\begin{tabular}{|c|c|c|c|c|c|c|}
\hline & \multicolumn{6}{|c|}{$T$ (mean)/B(mean) ratios } \\
\hline & \multicolumn{6}{|c|}{ NT } \\
\hline & \multicolumn{3}{|c|}{ 18F-FET-PET } & \multicolumn{3}{|c|}{ 18F-DPA-714 PET } \\
\hline & d14 vs d7 & d21 vs d7 & $\begin{array}{c}\text { d21 vs } \\
\text { d14 }\end{array}$ & d14 vs d7 & d21 vs d7 & d21 vs d14 \\
\hline Mean 1 & 1.46 & 2.03 & 2.03 & 2.43 & 2.43 & 2.43 \\
\hline Mean 2 & 1.33 & 1.33 & 1.46 & 2.43 & 2.17 & 2.17 \\
\hline Mean Diff. & 0.13 & 0.7 & 0.57 & 0 & 0.27 & 0.27 \\
\hline SE of diff. & 0.22 & 0.22 & 0.22 & 0.28 & 0.28 & 0.28 \\
\hline Adjusted P Value & 0.998 & 0.02 & 0.99 & $>0.9999$ & 0.97 & 0.97 \\
\hline \multirow[t]{4}{*}{ Significance } & ns & * & ns & $\mathrm{ns}$ & ns & ns \\
\hline & \multicolumn{6}{|c|}{ PLX5622 + Repopulation } \\
\hline & \multicolumn{3}{|c|}{ 18F-FET-PET } & \multicolumn{3}{|c|}{ 18F-DPA-714 PET } \\
\hline & d14 vs d7 & d21 vs d7 & $\begin{array}{c}\text { d21 vs } \\
\text { d14 }\end{array}$ & d14 vs d7 & d21 vs d7 & d21 vs d14 \\
\hline Mean 1 & 1.54 & 1.84 & 1.84 & 2.66 & 2.66 & 2.38 \\
\hline Mean 2 & 1.48 & 1.54 & 1.48 & 2.29 & 2.38 & 2.29 \\
\hline Mean Diff. & 0.06 & 0.3 & 0.37 & 0.37 & 0.28 & 0.09 \\
\hline SE of diff. & 0.099 & 0.11 & 0.11 & 0.16 & 0.16 & 0.16 \\
\hline Adjusted P Value & 0.998 & 0.076 & 0.02 & 0.22 & 0.56 & 0.992 \\
\hline Significance & ns & ns & * & ns & ns & ns \\
\hline
\end{tabular}

\begin{tabular}{|c|c|c|c|c|c|c|}
\hline & \multicolumn{6}{|c|}{$\mathrm{T}(\mathrm{max}) / \mathrm{B}$ (mean) ratios } \\
\hline & \multicolumn{6}{|c|}{ NT } \\
\hline & \multicolumn{3}{|c|}{ 18F-FET-PET } & \multicolumn{3}{|c|}{ 18F-DPA-714 PET } \\
\hline & d14 vs d7 & d21 vs d7 & $\begin{array}{c}\text { d21 vs } \\
\text { d14 }\end{array}$ & d14 vs d7 & d21 vs d7 & d21 vs d14 \\
\hline Mean 1 & 1.97 & 3.87 & 3.87 & 5 & 5 & 3.97 \\
\hline Mean 2 & 1.67 & 1.67 & 1.97 & 3.97 & 3.57 & 3.57 \\
\hline Mean Diff. & 0.3 & 2.2 & 1.9 & 1.03 & 1.43 & 0.4 \\
\hline SE of diff. & 0.34 & 0.34 & 0.34 & 1.27 & 1.27 & 1.27 \\
\hline Adjusted P Value & 0.98 & $<0.0001$ & $<0.0001$ & 0.99 & 0.91 & $>0.9999$ \\
\hline \multirow[t]{4}{*}{ Significance } & $\mathrm{ns}$ & $* * * *$ & $* * * *$ & $\mathrm{~ns}$ & $\mathrm{~ns}$ & $\mathrm{~ns}$ \\
\hline & \multicolumn{6}{|c|}{ PLX5622 + Repopulation } \\
\hline & \multicolumn{3}{|c|}{ 18F-FET-PET } & \multicolumn{3}{|c|}{ 18F-DPA-714 PET } \\
\hline & d14 vs d7 & d21 vs d7 & $\begin{array}{c}\text { d21 vs } \\
\text { d14 }\end{array}$ & d14 vs d7 & d21 vs d7 & d21 vs d14 \\
\hline Mean 1 & 2.18 & 2.47 & 2.47 & 5.42 & 5.42 & 4.34 \\
\hline Mean 2 & 1.96 & 1.96 & 2.18 & 4.18 & 4.34 & 4.18 \\
\hline Mean Diff. & 0.22 & 0.51 & 0.29 & 1.24 & 1.08 & 0.16 \\
\hline SE of diff. & 0.16 & 0.18 & 0.18 & 0.73 & 0.73 & 0.73 \\
\hline Adjusted P Value & 0.8 & 0.062 & 0.67 & 0.57 & 0.73 & $>0.9999$ \\
\hline Significance & ns & ns & ns & ns & ns & ns \\
\hline
\end{tabular}

\begin{tabular}{|c|c|c|c|c|c|c|}
\cline { 2 - 7 } \multicolumn{1}{c|}{} & \multicolumn{5}{c|}{ NT vs Repopulation 18F-FET-PET (Tmax/Bmean ratio) } \\
\cline { 2 - 7 } & Mean 1 & Mean 2 & Mean Diff. & SE of diff. & Adjusted P Value & Significance \\
\hline d21 vs d21 & 3.86 & 2.47 & 1.4 & 0.28 & 0.0001 & $* \star *$ \\
\hline
\end{tabular}

Abbreviation: NT: non treated; Gd-CE: Gadolinium contrast enhanced; T/B: tumor-to-background 


\section{Appendix}

\section{CSF-1R inhibition shows immune-modulatory effects on glioma-associated myeloid-derived cells}

A high frequency of polymorphnuclear (PMN)-MDSCs was detected in the TME of PLX5622-treated animals compared to the NT group (CD11 b+Gr1 ${ }^{+} \mathrm{Ly}_{6 \mathrm{G}}{ }^{+} \mathrm{Ly} 6 \mathrm{C}-23.2 \%$ vs $10.2 \%$ ); after repopulation, the number of PMNMDSCs was comparable to the NT group (CD11 $\mathrm{b}^{+} \mathrm{Gr} 1^{+} \mathrm{Ly} 6 \mathrm{G}^{+} \mathrm{Ly} 6 \mathrm{C}^{-}$: $\left.9.7 \%\right)$. On the contrary, monocytic (Mo)MDSCs decreased after PLX5622 treatment compared to the NT group, and they increased again after repopulation (CD11b+Gr1 ${ }^{+}$Ly6G Ly6C $C^{+}: 29.6 \%$ vs $34.7 \%$ and $35.5 \%$ respectively). In line with histology, GAMM were reduced especially after repopulation compared to the NT group (CD11b $\mathrm{br}^{+}$: $18.8 \%$ vs $24.4 \%$ ) (Supplemental Figure 8A). PLX5622 treatment decreased surface expression of CD68 and CD206, markers of an M2-like phenotype (CD11b $\left.{ }^{+} \mathrm{Gr} 1^{+} \mathrm{CD} 68^{+} \mathrm{CD} 206^{+}: 23.6 \%\right)$ and the effect was maintained after repopulation (19.1 \%), in particular in the case of PMN-MDSCs (Gr1 ${ }^{\text {high }} \mathrm{CD}^{+} 8^{+} \mathrm{CD} 206^{+}: 29.8 \% \mathrm{NT}, 5.6 \%$ PLX5622, 0\% repopulation). Furthermore, PLX5622 treatment and subsequent repopulation affected the expression levels of MHC class II, a marker of activation and differentiation. MHC-II expression was increased in Mo-MDSCs after treatment and repopulation ( $\mathrm{Gr} 1^{\text {int }} \mathrm{Ly} 6 \mathrm{C}^{+} \mathrm{MHC} \mathrm{II}^{+}: 29.9 \%$ and $29.6 \%$ respectively). On the contrary, it was significantly decreased in PMN-MDSCs after repopulation resulting in a population 100\% MHC II negative. Looking at the expression of MHC-II on GAMM, the treatment decreased the marker levels, but they increased again after repopulation (CD11b ${ }^{+}$Gr1 CD68 ${ }^{+} \mathrm{MHC} \quad \mathrm{II}^{+}: 52.9 \%$ and $77.1 \%$ respectively) (Supplemental Figure 8B). Finally, PLX5622 immunomodulation affected TSPO expression confirming the imaging and histological results. Mo-MDSCs and PMN-MDSCs were characterized by reduced TSPO expression after PLX5622 treatment (Gr1 ${ }^{\text {int }}$ Ly6C $^{+}$TSPO $^{+}: 8.7 \%$ and Gr1 $\left.{ }^{\text {high }} \mathrm{Ly6G}^{+} \mathrm{TSPO}^{+}: 11.1 \%\right)$ with PMN-MDSCs negative for TSPO after repopulation. TSPO expression on GAMM was reduced after treatment and increased again during repopulation (CD11 $\mathrm{b}^{+} \mathrm{Gr}^{-} \mathrm{TSPO}^{+}: 15.7 \%$ and $29.4 \%$ respectively) (Supplemental Figure 8C). 
A Repopulation study

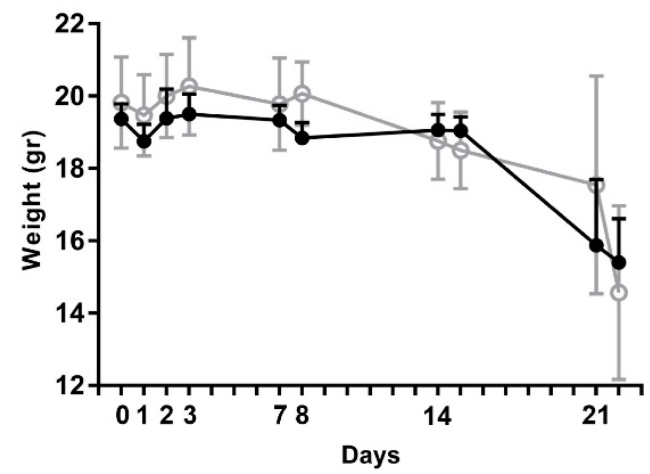

$\rightarrow$ NT

๑- PLX5622 + Repop.

B

Preconditioning study

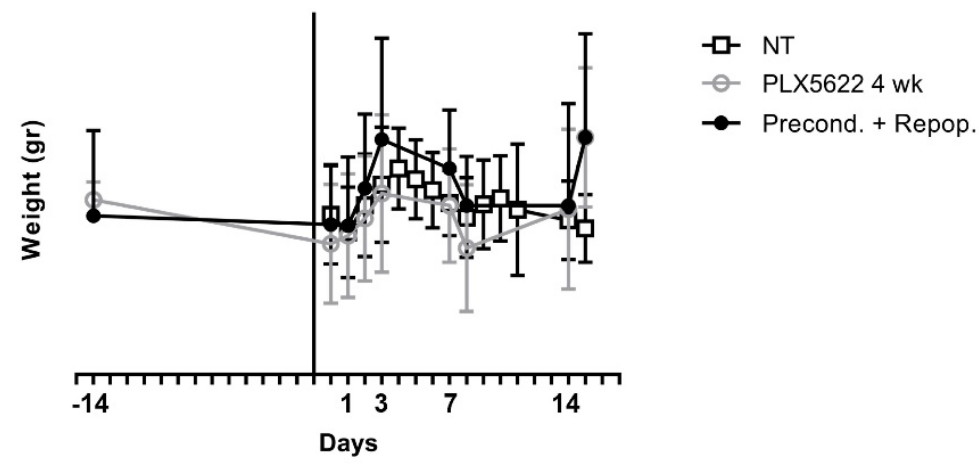

Supplemental Figure 1. Weight curves to monitor possible variations due to PLX5622 treatment. (A) Weight curve for the repopulation study and (B) for the preconditioning study. 

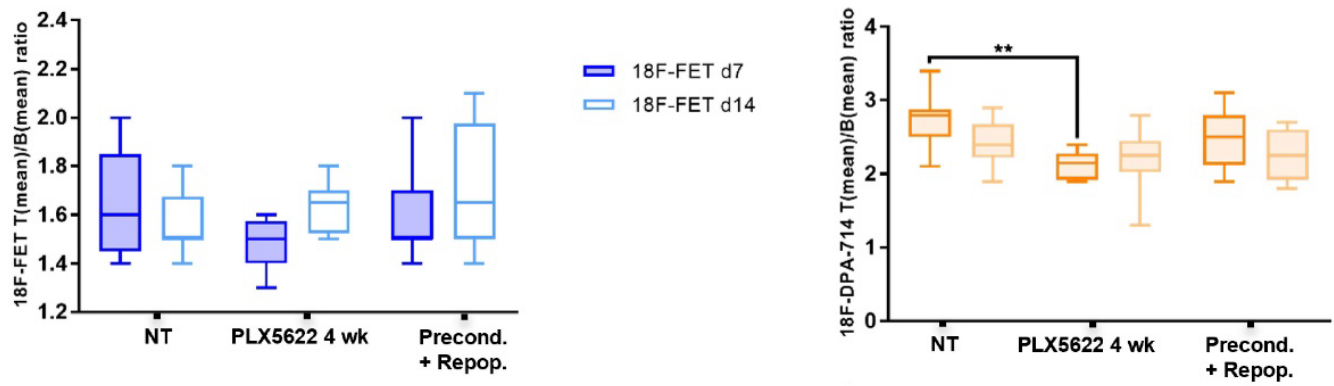

다-DPA-714 d7

18 F-FET d7

18F-FET d14

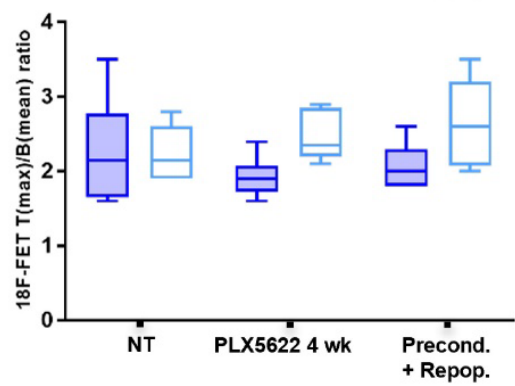

ש 18F-FET d7

$\square$ 18F-FET d14

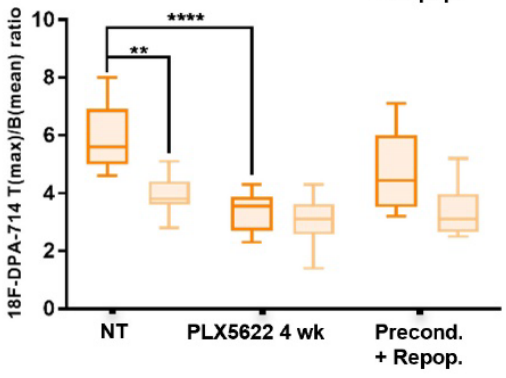

— 18F-DPA-714 d7

12 18F-DPA-714 d14

Supplemental Figure 2. Additional quantitative analysis of 18F-FET and 18F-DPA-714

T/B uptake ratio in the preconditioning model.

Abbreviations: T/B: tumor-to-background; NT: non treated. 


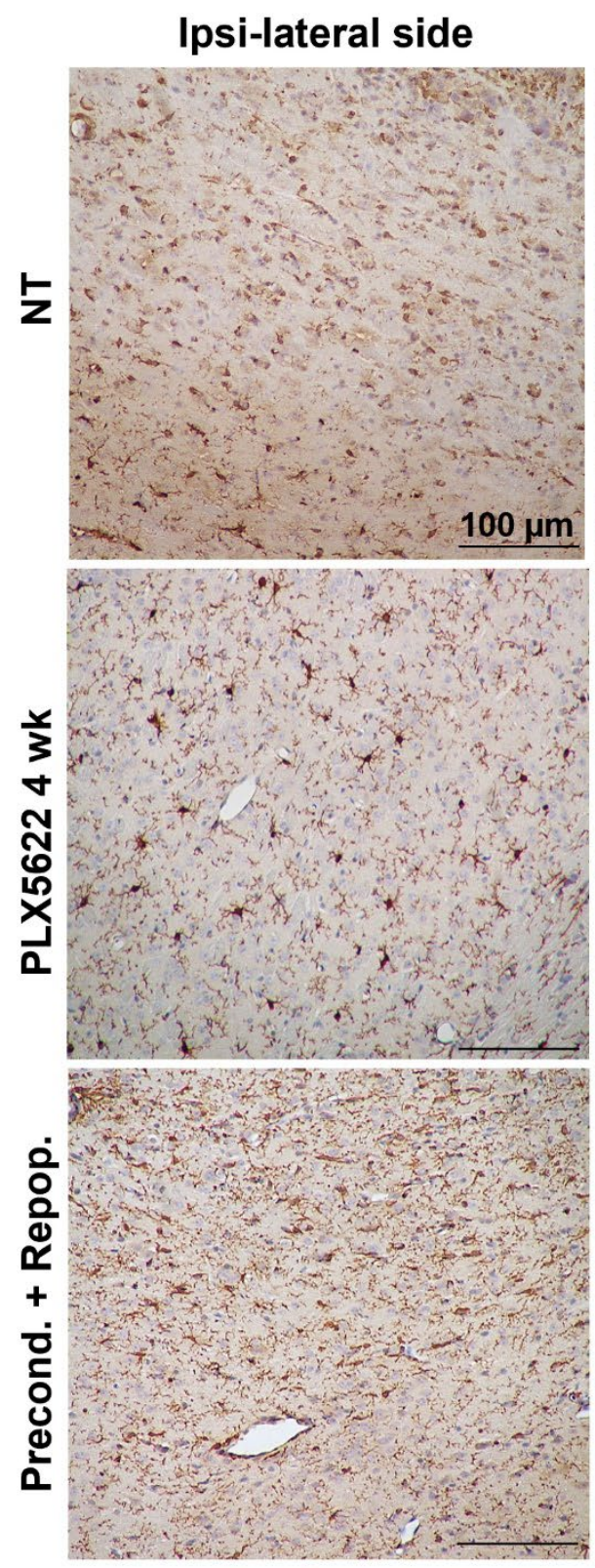

Contra-lateral side
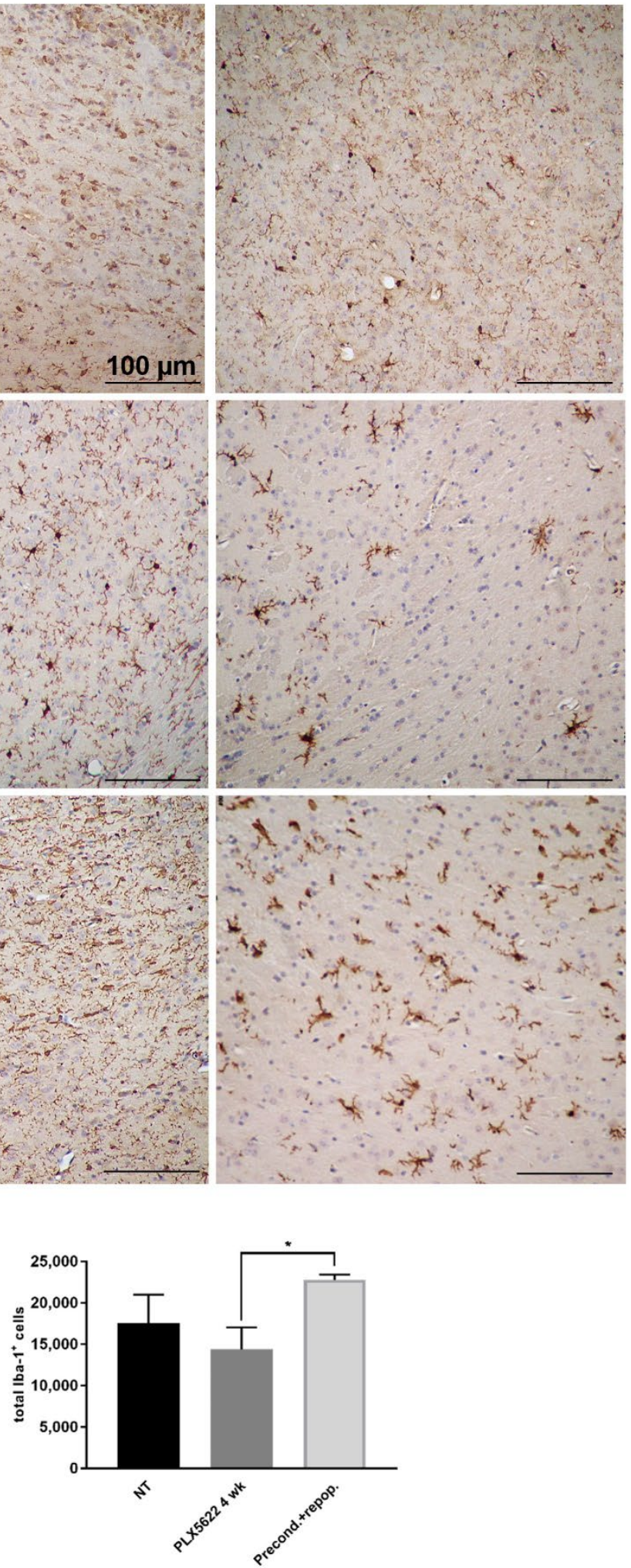

Supplemental Figure 3. Therapy-resistant $\mathrm{Iba} 1^{+}$cells were detected in the ipsi- and contralateral hemispheres after CSF-1R chronic inhibition. Iba1 labeling of histological samples from gliomabearing mice (NT, chronically-treated and preconditioned and repopulated), and quantification of Iba $1^{+}$cells. ( $N=3$ /group). * $p \leq 0.05$. CSF-1R: colony stimulating factor-1 receptor; NT: non-treated. 

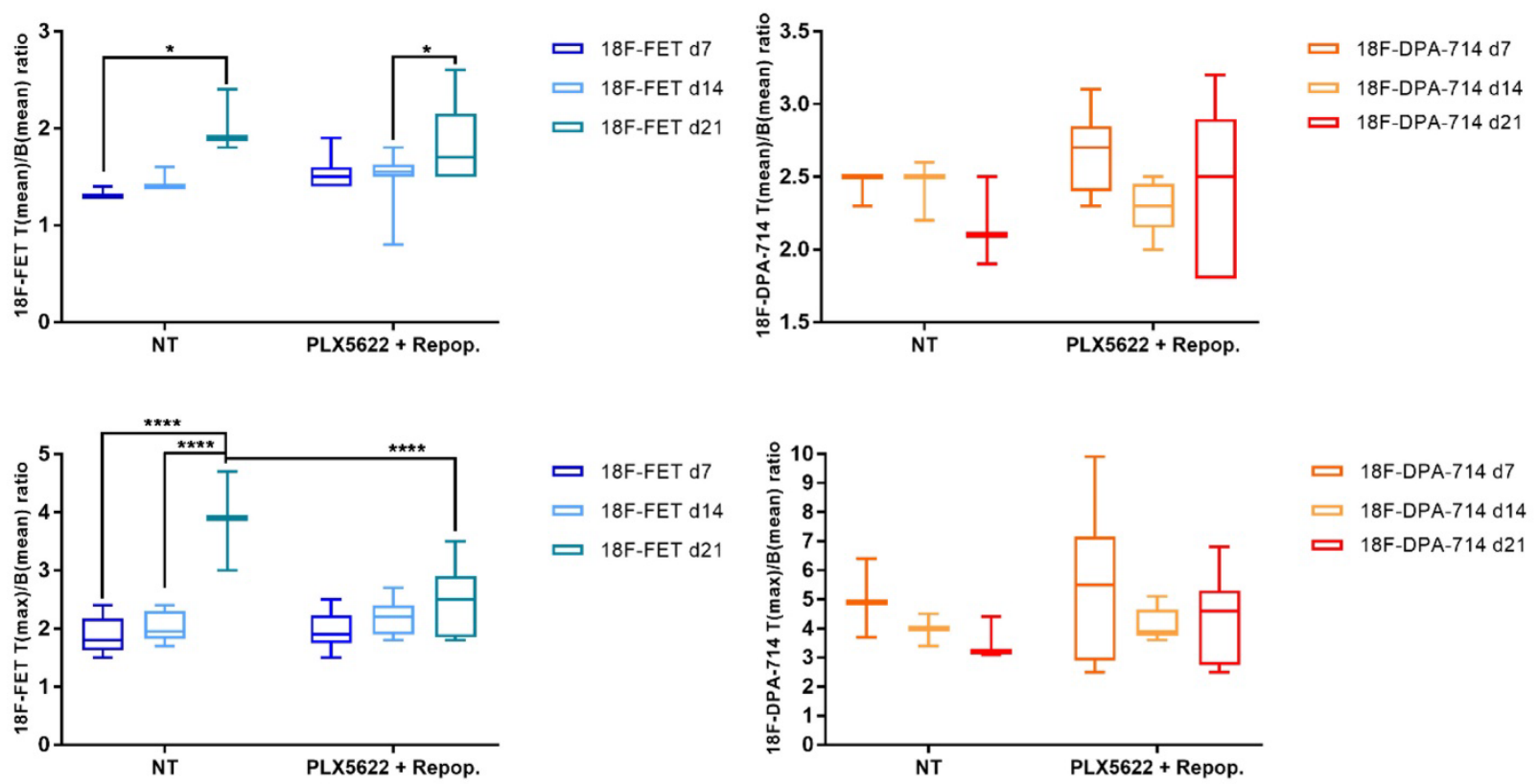

Supplemental Figure 4. Additional quantitative analysis of 18F-FET and 18F-DPA714 T/B uptake ratio in the repopulation model.

Abbreviations: T/B: tumor-to-background; NT: non treated. 


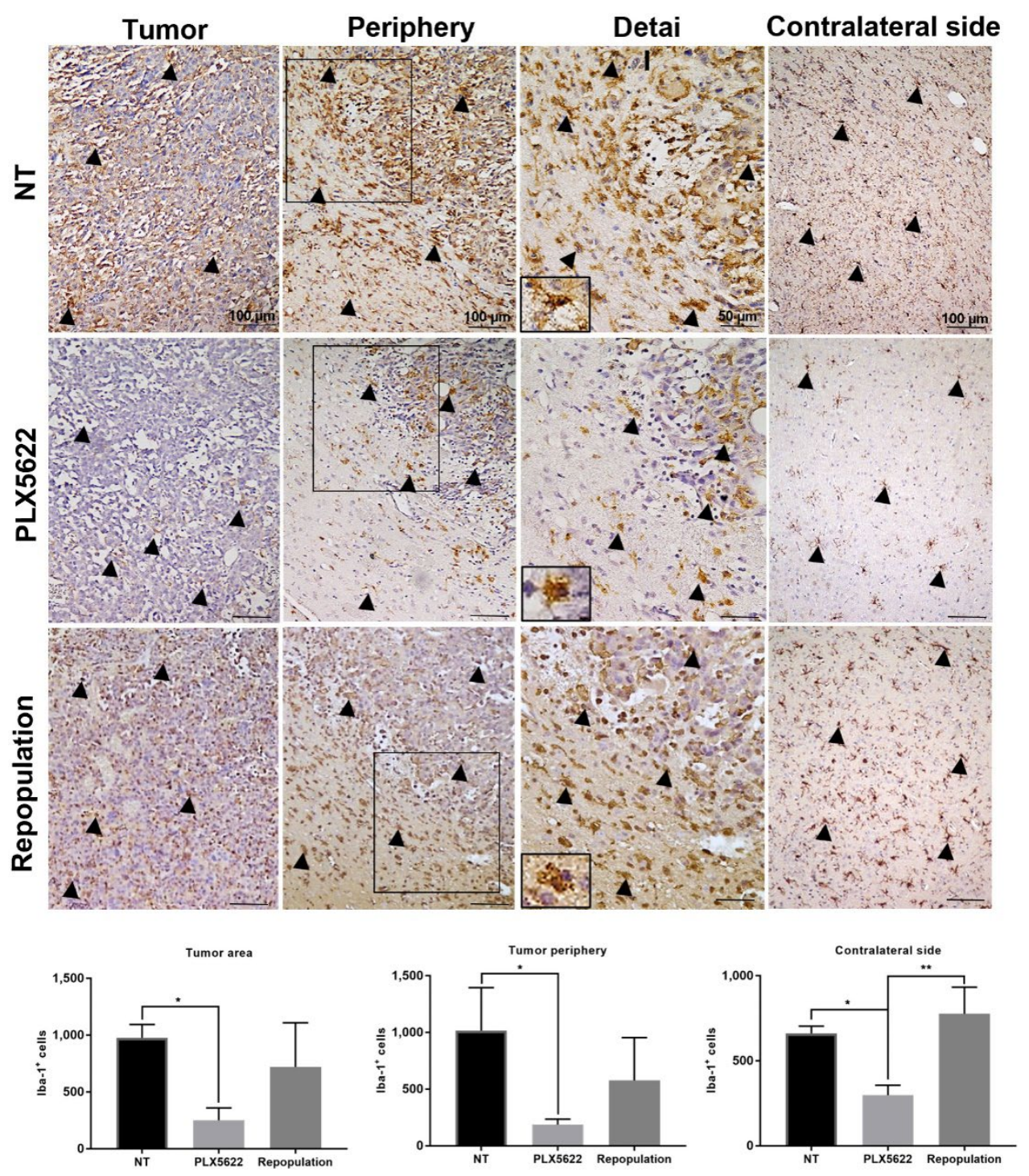

Supplemental Figure 5. Analysis of Iba1 immunoreactivity to investigate PLX5622mediated GAMM depletion and repopulation. Detection of $\mathrm{Iba} 1^{+}$cells in histological samples from glioma-bearing mice, NT, PLX5622-treated and repopulated with quantification of $\mathrm{Iba} 1^{+}$cells in the tumor area, tumor periphery and contralateral side ( $n=3$ /group). Arrows: representative $\mid \mathrm{Iba}{ }^{+}$cells. Squares: detailed images. Small squares: magnified details. ${ }^{*} p \leq 0.05 ;{ }^{* *} p \leq 0.01 ;{ }^{* * *} p \leq 0.001$. GAMM: gliomaassociated microglia/macrophages; NT: non-treated. 

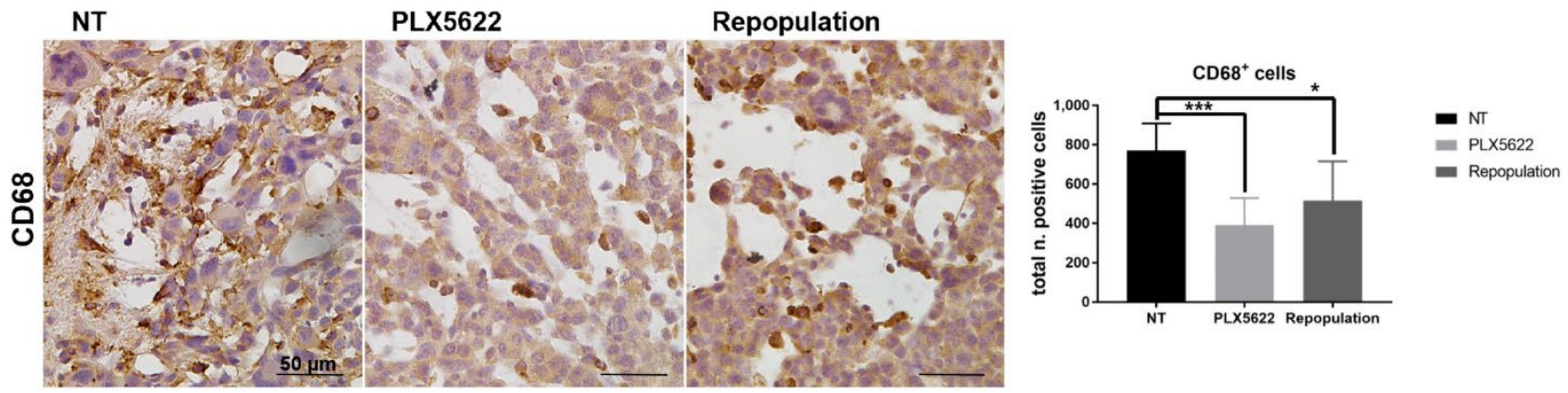

Supplemental Figure 6. Analysis of CD68 immunoreactivity. Paraffin embedded tissues labeled for CD68 in NT, PLX5622-treated and repopulated animals with the respective quantification of $C D 68^{+}$cells. ( $n=3$ /group). ${ }^{*} p \leq 0.05 ;{ }^{* *} p \leq 0.01 ;{ }^{* * *} p \leq$ 0.001. 


\section{Repopulation study}
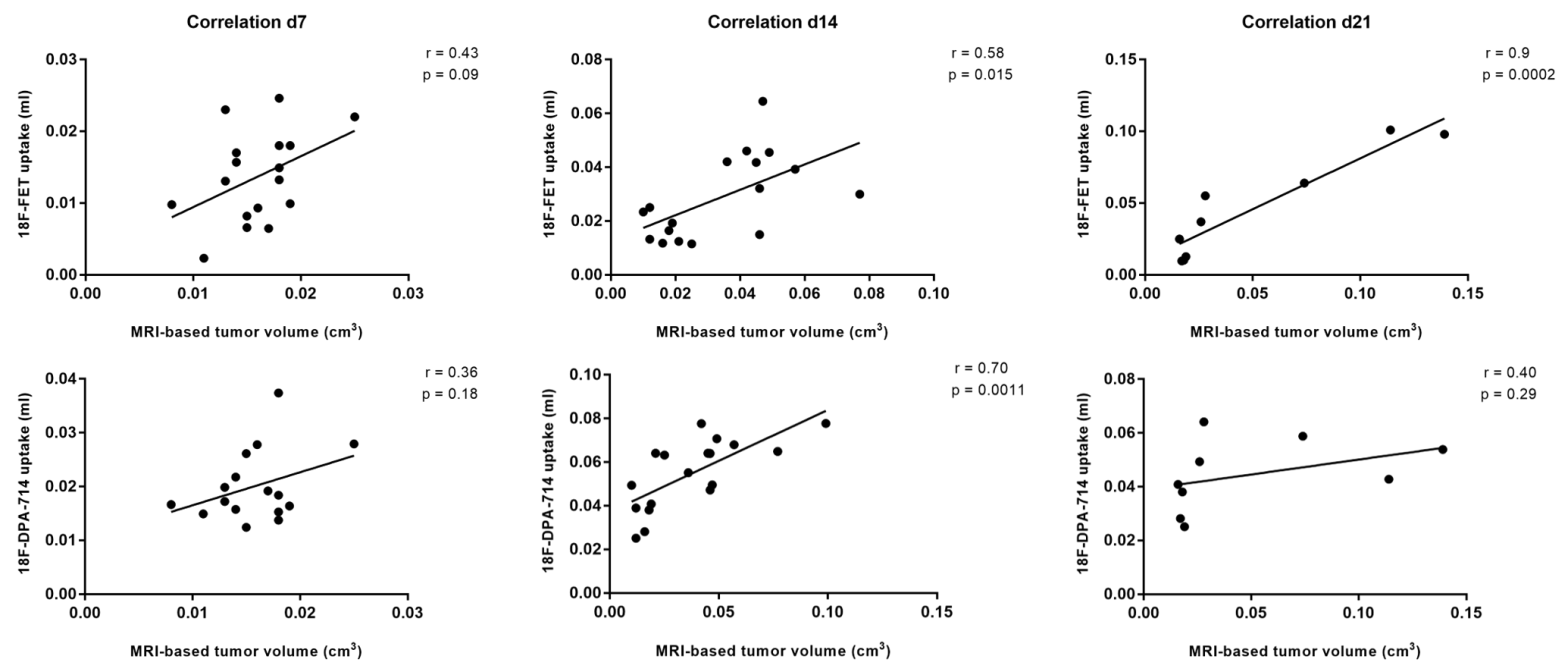

Supplemental Figure 7. Analyses of the correlation between MRI-based TV and $\left[{ }^{18} \mathrm{~F}\right] \mathrm{FET}$ or $\left[{ }^{18} \mathrm{~F}\right] \mathrm{DPA}-714$ uptake. Correlation of MRI-based TV with $\left[{ }^{18} \mathrm{~F}\right] \mathrm{FET}$ and $\left.{ }^{18} \mathrm{~F}\right] \mathrm{DPA}-714$ uptake in the PLX5622 group before and after treatment.

Abbreviations: TV: tumor volume; CE-MRI: contrast-enhanced magnetic resonance imaging. 
A
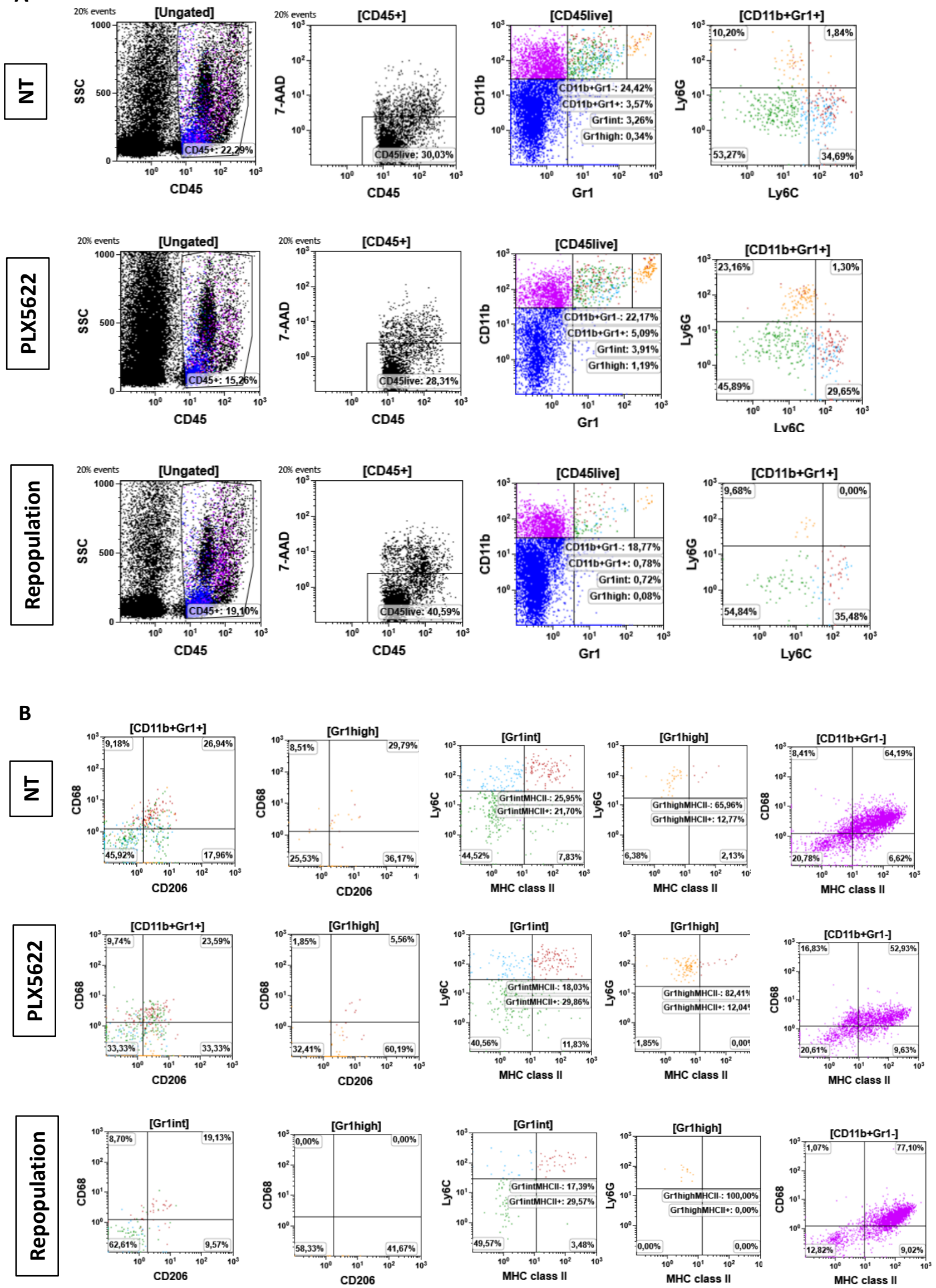
C
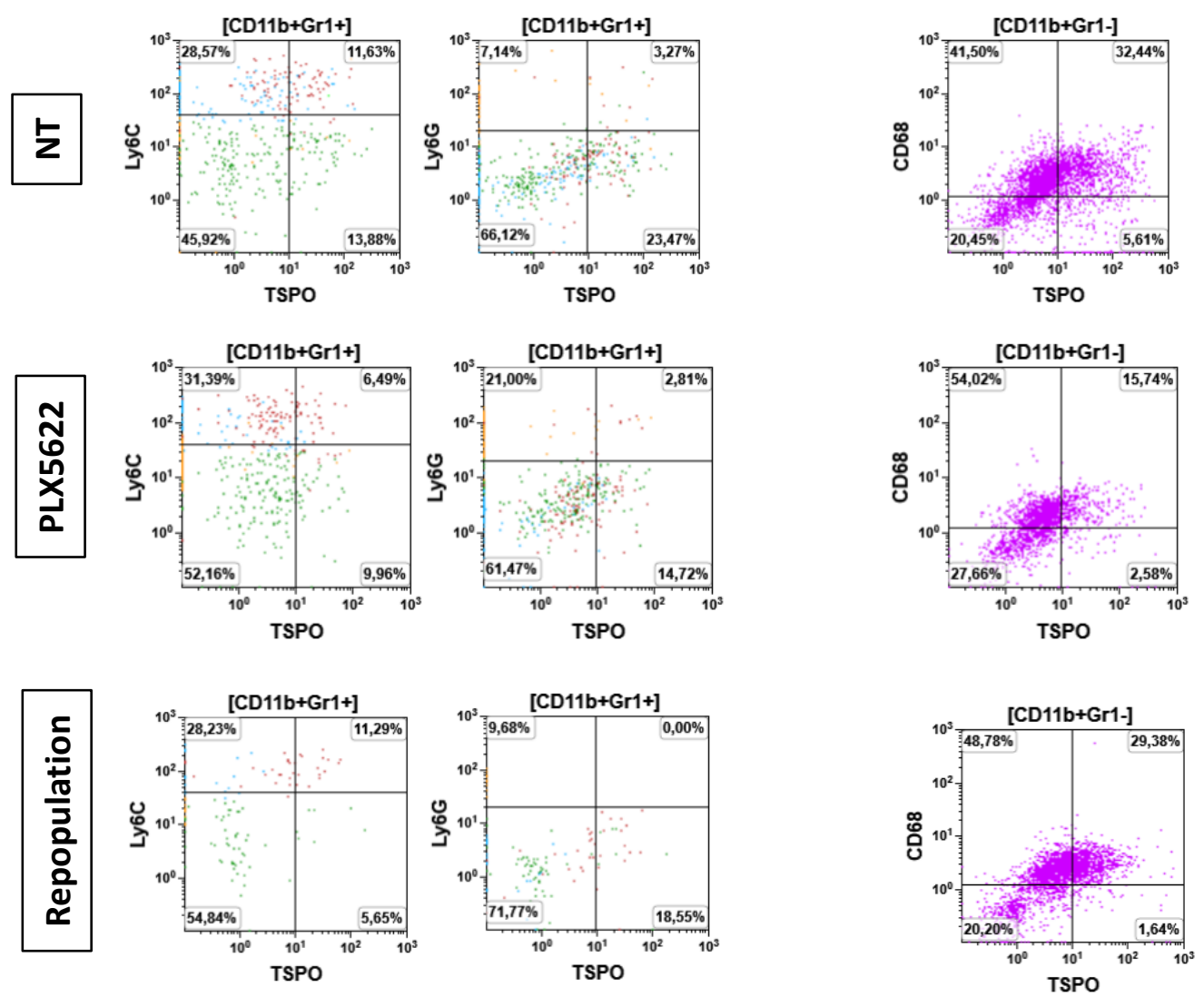

Supplemental Figure 8. Multiparametric flow cytometry analyses show CSF-1R immune-modulatory effects in the TME. Cell sorting and gating protocol adopted for the analyses of NT, PLX5622-treated group and the repopulation group focusing on (A) the myeloid-derived cell compartment, (B) the expression of CD68, CD206 and MHC class II markers and (C) the expression of TSPO.

Abbreviations: NT: non-treated; MHC: major histocompatibility complex; TSPO: translocator protein 